\title{
Relation of polar auroral arcs to magnetotail twisting and IMF rotation: a systematic MHD simulation study
}

\author{
A. Kullen ${ }^{1}$ and P. Janhunen ${ }^{2}$ \\ ${ }^{1}$ Alfvén Laboratory, Royal Institute of Technology, Stockholm, Sweden \\ ${ }^{2}$ Geophysical Research, Finnish Meteorological Institute, Helsinki, Finland
}

Received: 21 August 2002 - Revised: 4 July 2003 - Accepted: 22 July 2003 - Published: 19 March 2004

\begin{abstract}
We investigate with the help of a magnetohydrodynamic (MHD) model how the large-scale topology of the magnetosphere develops for a constant interplanetary magnetic field (IMF) with different IMF clock angles and for an IMF $B_{y}$ sign change during northward IMF. A detailed examination of the topological changes in the tail and the ionosphere for different IMF conditions shows a good agreement with observational results.

The MHD simulations for different constant IMF clock angle cases show the expected field-line bending and tail twisting for nonzero IMF $B_{y}$. The tail becomes longer and at its tailward end stronger twisted for IMF $B_{z}>\left|B_{y}\right|$ than for IMF $B_{z}<\left|B_{y}\right|$. The field lines originating in the high-latitude flank of the far-tail plasma sheet map into the near-Earth tail lobes and to a strongly poleward displaced polar cap boundary. A comparison with observations suggests that an ovalaligned arc may occur on the high-latitude part of the polar cap boundary.

An IMF $B_{y}$ sign change causes large deformations of the tail. After the IMF $B_{y}$ flip the near-Earth and far-tail plasma sheet regions are oppositely twisted which causes in the nearEarth tail a bifurcation of the closed field line region that moves from one flank to the other. The bifurcated part of the closed field line region maps to a bridge of closed field lines moving over the entire polar cap. This moving bridge may be interpreted as the mapped region of a moving transpolar arc. Based on earlier observations, such a type of polar arcs is expected to occur after an IMF $B_{y}$ sign change.
\end{abstract}

Key words. Ionosphere (auroral ionosphere; ionospheremagnetosphere interactions). Space plasma physics (numerical simulation studies)

\section{Introduction}

The structure of the magnetosphere is strongly controlled by the direction of the interplanetary magnetic field (IMF). Especially the north-south component of the IMF, but also the

Correspondence to: A. Kullen

(anita.kullen@alfvenlab.kth.se) dawn-dusk component of the IMF influences the auroral precipitation pattern in the ionosphere and the large-scale topology of the magnetotail.

1.1 IMF $B_{y}$ and $B_{z}$ effects on the auroral precipitation pattern

It is well known that the polar cap expands during southward IMF conditions and contracts during northward IMF. Makita et al. (1988) showed that the thickness of the auroral oval increases and the size of the polar cap decreases with the strength of northward IMF. Polar auroral arcs (auroral arcs occurring poleward of the auroral oval) are a common phenomenon occurring during northward IMF (Valladares et al., 1994, and references therein).

A recent statistical study by Kullen et al. (2002) about the dependence of large-scale polar arcs on the IMF direction distinguishes between five types of polar arcs which are shown each to be related to a characteristic combination of solar wind parameters. The results show that those polar arcs occurring after $1-2 \mathrm{~h}$ of nearly constant IMF with a positive IMF $B_{z}$ and a nonzero IMF $B_{y}$ component are always static polar arcs near one oval side (oval-aligned arcs). Ovalaligned arcs occur for a broad range of time spans. On an average, they last for $2 \mathrm{~h}$. Polar arcs moving from one oval side to the other (moving arcs) can in most cases be related to a large-scale sign change of IMF $B_{y}$ which takes place up to one hour before the arc's appearance. Moving arcs are usually transpolar arcs, connecting the nightside auroral oval with the dayside one. IMF $B_{z}$ is predominantly positive one to two hours before and during a moving arc event. Moving arcs last between one and four hours, unless a large substorm occurs or the IMF changes to a persistently southward orientation. Such conditions lead to the disappearance of all types of polar auroral arcs. There exist other polar arc formations that have hardly been reported in literature: Immediately after an IMF $B_{z}$ sign change often a short-lived polar arc occurs which bends into the polar cap with the anti-sunward end fixed to the oval boundary (bending arc). In rare cases, one single arc rises from the nightside oval boundary towards 
noon (midnight arc). This type of arc always appears at the very end of a substorm recovery phase. Other polar arc events are more complicated and involve three or more arcs separating from the oval-sides or the nightside oval boundary (multiple arc events).

The location of polar arcs is strongly dependent on the sign of IMF $B_{y}$. A statistical study about polar cap size and location during northward IMF by Elphinstone et al. (1990) shows that the location of polar arcs is strongly correlated to the IMF $B_{y}$ component, while the main oval keeps its circular shape. Most dawnside oval-aligned arcs occur for negative IMF $B_{y}$ and all dusk side arcs occur for positive IMF $B_{y}$. Moving polar arcs have been found to separate from the dawn (dusk) side of the oval after an IMF $B_{y}$ sign change from negative to positive (positive to negative) (Cumnock et al., 1997; Chang et al., 1998). The statistics of Kullen et al. (2002) confirms these results: In the Northern Hemisphere, all arc types that include a poleward motion (moving and bending arcs) move toward the side of the oval pointed at by IMF $B_{y}$, whereas the non-moving arcs (oval-aligned and midnight arcs) are located at that side of the oval from the very beginning.

\subsection{Polar arc models}

As large-scale polar auroral arcs are commonly believed to have their source region in the tail plasma sheet (Frank and Craven, 1988), they probably lie on closed field lines. The occurrence of closed field lines poleward of the main auroral oval has to be addressed by any model explaining large-scale polar arcs.

A model, first proposed by Meng (1981), interprets polar arcs occurring during nonzero IMF $B_{y}$ as the poleward boundary of an expanded auroral oval. A highly contracted polar cap occurs typically during strongly northward IMF. A further poleward displacement of the polar cap boundary on one oval side is caused by a twisting of the tail plasma sheet (Cowley, 1981; Kullen and Blomberg, 1996). A twist of the magnetotail is well-known to be connected to the IMF $B_{y}$ component (Kaymaz et al., 1994, and references therein). The hypothesis of a polar arc being the poleward part of the auroral oval is best suited as an explanation for oval-aligned arcs, as these are static arcs that are often not greatly displaced from the oval boundary.

Moving polar arcs are during part of their lifetime located in the middle of the polar cap near the noon-midnight meridian. It has been suggested by several authors (e.g. Kan and Burke, 1985; Frank et al., 1986; Chang et al., 1998) that during such an event the magnetotail plasma sheet is bifurcated, i.e. a part of the plasma sheet extends to very highlatitudes, separating the lobes into a dawn and a duskward part. Huang et al. (1989) suggested that filamentary extensions of the plasma sheet may reach into the lobes.

Kullen (2000) proposed a model that does not include a plasma sheet bifurcation. Instead, it is suggested that the evolution of a moving arc may be related to a rotation of the magnetotail twist which is initiated by an IMF $B_{y}$ sign change. To test this idea, the T89 (Tsyganenko, 1989) model has been modified in such a way that the near-Earth and the far-tail regions are oppositely twisted and for each time step the twist rotation is located further tailward. Mapping from the tail current sheet to the ionosphere results in a bar of closed field lines separating from the main oval and moving poleward for a tailward propagation of the tail twist rotation. This bar of closed field lines is interpreted as the mapped region of a moving polar arc. The model polar arc disappears when the rotation of the tail is completed and the entire tail has returned to a topology typical for uniform nonzero IMF $B_{y}$. Varying the input parameters in the model indicates that the duration of a polar arc event is dependent on the magnitude of IMF $B_{y}$, but not on the spatial extent of the IMF $B_{y}$ sign reversal region. A broad dawn-duskward extension of the model polar arc appears for a high IMF $B_{y}$ magnitude and/or a long sign reversal period. An important result is the finding that although the plasma sheet (as identified from the $B^{2}$ isocontour lines) is forced ad hoc to keep a butterfly shape, the region of closed field lines is bifurcated in the near-Earth tail. The reason for the bifurcation is that closed field lines which have their turning point near the high-latitude flank of the far-tail plasma sheet, map high up into the lobes of the oppositely twisted near-Earth tail (for a 3-D-schematic of the resulting tail topology, see Fig. 2b in Kullen et al., 2002). The Kullen (2000) polar arc model is entirely based on the assumption that an IMF $B_{y}$ sign change causes first the near-Earth tail and later the far tail to respond such that in an intermediate state the near-Earth and the fartail regions are oppositely twisted. Data and/or magnetohydrodynamic (MHD) studies are necessary to investigate the correctness of this assumption.

A polar arc model that became widely accepted in recent years has been developed by Newell et al. (1997) and Chang et al. (1998). It explains the creation of a new region of open field lines dawnward (or duskward) of the main polar cap near noon with an abrupt jump in the dayside merging line. It is proposed that this jump is caused by an IMF $B_{z}$ or IMF $B_{y}$ sign change. The closed field line bridge in the ionosphere between the old and the new region of open field lines is supposed to be the location where a polar arc occurs. Chang et al. (1998) predicted that several sign changes would be necessary for a polar arc to exist for a longer time. However, observations show that long-lasting, moving polar arcs exist where no further IMF sign changes occur after a large-scale IMF $B_{y}$ sign reversal (Cumnock et al., 2002). As the model includes the immediate occurrence of a polar arc after an IMF sign change and a short lifetime of the arc in case that no further IMF variations occur, we believe that this model is a better fit as an explanation for bending arcs than for the long-lasting moving arcs.

Many models from the 1980's assume that transpolar arcs typically grow from the nightside oval towards noon (e.g. Kan and Burke, 1985; Frank et al., 1986). However, a large number of observational papers show that most arcs separate from the dawn or dusk side of the oval (e.g. Chang et al., 1998). Single midnight arcs are extremely rare events and 
occur only after a period of predominantly strong northward IMF at the end of a substorm recovery phase (Kullen et al., 2002). They all develop from a strongly contracted poleward boundary of the nightside auroral oval. In the midnight arc model by Rezhenov (1995) precisely these characteristics are suggested as preconditions for midnight arcs to occur. He suggests that an interchange instability may occur at the tailward end of a short plasma sheet that causes a tongue of plasma to move tailward. The tailward moving plasma sheet tongue is supposed to map to a closed field line bar that grows from midnight to noon in the polar cap.

1.3 IMF $B_{y}$ and $B_{z}$ effects on the magnetotail: observations

The effect of the IMF $B_{y}$ component on the magnetotail consists mainly in a dawn-duskward bending of the magnetic field lines and a twist of the entire tail. The field-line bending is connected to an additional $B_{y}$ field that appears in the entire tail. As this extra $B_{y}$ term has the same direction and a magnitude at a fraction of the IMF $B_{y}$ value, it has been often referred to as "IMF $B_{y}$ penetration field", which indicates a physical process that has not been proven to take place in the magnetosphere. For simplicity, we use this term as well when describing previous observational results, but only as a description of the rate of an extra $B_{y}$ term occurring in the tail.

From numerous observations it is known that the IMF $B_{y}$ penetration field is nonuniform. The average $B_{y}$ penetration in the tail lies around 10 to $14 \%$ of the total IMF $B_{y}$ magnitude (e.g. Fairfield et al., 1979; Cowley, 1981; Kaymaz et al., 1994). A statistical study of the mid-tail region by Kaymaz et al. (1994) confirms earlier findings that the IMF $B_{y}$ penetration is much stronger in the plasma sheet than in the lobes. They found on average, 9 and $26 \%$ penetration in the lobes and in the plasma sheet, respectively. A maximum penetration of $35 \%$ was discovered near the plasma sheet flanks. The strongest IMF $B_{y}$ penetration into the tail seems to occur at the neutral sheet (defined as the location of the magnetic field line reversal from tailward to earthward, i.e. where $B_{x}$ changes sign). Lui (1986) and Sergeev (1987) reported penetration rates of 50 and $60 \%$ of the total IMF $B_{y}$ field, and Borovsky et al. (1998) found an even higher penetration rate (76\%) for cases with $30 \mathrm{~min}$ or more constant IMF $B_{y}$ before the neutral sheet crossing of the satellite. All results so far mentioned refer to a tail region of about 20 to $30 R_{E}$ downtail from the Earth.

In many data studies, a twisting of the current sheet by 6 to 18 degrees for nonzero IMF $B_{y}$ was found (e.g. Sibeck et al., 1986; Tsyganenko, 1998; Kaymaz et al., 1994). Kaymaz et al. (1994) showed that, on average, the current sheet is more twisted near the flanks than in the central tail, and Tsyganenko (1998) found the tail twist to increase with the distance from the Earth. MacWan (1992) reported twists over 90 degrees in the distant magnetotail $\left(x=115-220 R_{E}\right)$. In a statistical magnetotail study by Owen et al. (1995) much lower twists are found, on average, but according to the au- thors large twists of about 50-60 degrees are possible in individual cases. One major result of the study by Owen et al. (1995) is a correlation between the degree of plasma sheet twisting and the sign of IMF $B_{z}$. For northward IMF, plasma sheet twists are, on average, twice $\left(13^{\circ}\right.$ for $B_{y}<0$ and $24^{\circ}$ for $\left.B_{y}>0\right)$ those for southward IMF ( $6^{\circ}$ for $B_{y}<0$ and $12^{\circ}$ for $B_{y}>0$ ). The observation of a high degree of twisting for low geomagnetic activity is probably due to the connection between quiet conditions and northward IMF.

It seems hard to define the length of the closed field line region from observations. MacWan (1992) reported that only for dynamic topology changes the region of closed field lines reaches as far downtail as the region of his measurements. In the static cases the last closed field lines are earthward of $115-220 R_{E}$.

1.4 IMF $B_{y}$ and $B_{z}$ effects on the magnetosphere: MHD model results

Many MHD simulations have been performed to study the magnetospheric topology during purely northward IMF. In most MHD models the tail becomes completely closed for persistently northward IMF (e.g. Fedder and Lyon, 1995; Song et al., 1999). In this case, the tail length has been found to be inversely proportional to the strength of positive IMF $B_{z}$ (e.g. Song et al., 1999, and references therein).

When the tail is not closed, it contains open field lines connected with the IMF and it has no defined length. Instead, the tailward extension of the closed field line region is studied. Gombosi et al. (1998), using the same MHD model as Song et al. (1999), found that the closed field line region extends to $120-140 R_{E}$ during the first hour after an IMF $B_{z}$ northward turning and then shrinks to a constant length of about $60 R_{E}$. They concluded that a very long closed field line region for northward IMF seems only to appear as a transient phenomenon. Raeder et al. (1995) reported for purely northward IMF, a much shorter closed field line region of about $20-40 R_{E}$ near the $x$-axis but stretching further tailward near the flanks. The plasma population remains apparently the same earthward and tailward of the last closed field line (Song et al., 1999). The dawn-dusk diameter of the far-tail lobes depends on IMF $B_{z}$, being more narrow during northward IMF than during southward IMF (Raeder et al., 1995)

The IMF $B_{y}$ effects on the magnetotail as found from observations appear in many MHD simulations. The first simulation results focusing on IMF $B_{y}$ effects were published by Brecht et al. (1981). A run with constant south-dawnward IMF input was presented which shows a twist of the entire tail and an IMF $B_{y}$ penetration into the neutral sheet. Kaymaz et al. (1995) used the Fedder-Lyon MHD model (Fedder and Lyon, 1995) to compare their statistical results (Kaymaz et al., 1994) with MHD simulations. Both the IMF $B_{y}$ penetration and the twisting of the current sheet in the simulation are in good agreement with the observations. Simulations for constant IMF input presented by Ogino (1986) show clearly (although not discussed in further detail by the authors) that 
the degree of plasma sheet twisting during nonzero IMF $B_{y}$ is dependent on the sign of IMF $B_{z}$.

There exist several papers presenting MHD simulation results for a changing IMF clock angle $\theta\left(\theta=0^{\circ}\right.$ for northward, $\theta=90^{\circ}$ for dawnward or duskward, $\theta=180^{\circ}$ for southward IMF). Berchem et al. (1998) using the code described by Raeder et al. (1995) compared satellite data from the tail during a period of changing IMF with simulation results. In their simulations the closed field line region is long and the plasma sheet is highly twisted for small IMF clock angles only, as expected from the statistical study by Owen et al. (1995). The northern and southern open field line regions for IMF $\left|B_{y}\right|>B_{z}$ are well separated from each other in the far-tail $y z$-plane but for IMF $\left|B_{y}\right|<B_{z}$ they are more close to each other. A detailed study about a changing IMF clock angle is presented by Walker et al. (1999) using the MHD code developed by Ogino et al. (1992). They found, like Berchem et al. (1998), the tail twisting to be stronger for northward than for southward IMF. Moreover, they showed that the far tail is more strongly twisted than the near-Earth tail, in agreement with Tsyganenko's (1998) statistical results. In the simulations by Walker et al. (1999) the near-Earth tail responds first to an IMF change. The tail changes its twist first at the flanks, then in the central tail. A magnetopause reconfiguration in response to an IMF change takes up to $15 \mathrm{~min}$, the plasma sheet twist occurs 45-60 min after an IMF change reaches the dayside magnetopause. The near-Earth neutral line (NENL) at $x=20 R_{E}$ disappears within $45 \mathrm{~min}$ after a northward turning of the IMF and the region of closed field lines increases in length for a decreasing IMF clock angle. Slinker et al. (2001) compared polar cap observations by the Eureka dawn-dusk meridian scanning photometer (MSP) with simulation results of the Fedder-Lyon MHD model for an IMF $B_{y}$ sign change during mainly northward IMF. Their results basically confirm the model suggested by Kullen (2000). In the simulations a region of closed field lines moves over the entire polar cap, while the tail is changing its twist from one direction to the other. The closed field line bridge in the model polar cap corresponds to a dawn-duskward moving strip of auroral emissions seen in the MSP, which is most likely the signature of a large-scale moving polar arc.

\subsection{IMF $B_{y}$ and $B_{z}$ effects on the magnetosphere: theories}

Russell (1972) was first to predict a twisting of the magnetotail caused by the asymmetry of the reconnection between solar wind and magnetosphere during nonzero IMF $B_{y}$. He even predicted a stronger twisting during northward IMF due to possible lobe reconnection. Cowley (1981) argued in the same way. He proposed that the asymmetric addition of magnetic flux to the tail lobes from dayside reconnection during nonzero IMF $B_{y}$ conditions may result in a penetration of the IMF $B_{y}$ into the tail. With a simple model he showed how an assumed $B_{y}$ penetration field that decreases towards lower latitudes leads to a twisting of the tail lobe field around the $x$-axis. Neither the occurrence of the IMF $B_{y}$ penetration field in the region of closed field lines nor the plasma sheet twisting are explained in this paper, although Cowley mentioned, that both effects are probable to occur. Owen et al. (1995) proposed that the stronger tail twist during northward than during southward IMF is associated with lobe reconnection. Open field lines reconnected poleward of the northern cusp with the IMF map to the solar wind field lines south of the equatorial plane where they are frozen into the solar wind flow. Hence, these open field lines exert a much stronger torque on the tail than in the southward case. During southward IMF the field lines reconnect at the dayside magnetopause and are bent into the direction of IMF $B_{y}$, but remain in the same hemisphere. The field-line topology for a 45-degree IMF clock angle has been confirmed in MHD simulations by Tanaka (1999). From these models, it remains unclear how the twisting of the open field line regions causes a twisting of the tail plasma sheet, and how the IMF $B_{y}$ penetration field occurs in the region of closed field lines, being even stronger than in the lobes. The nonuniformity of the $B_{y}$ penetration field has been addressed by Voigt and Hilmer (1987). They showed with a 2-D-MHD simulation that, assuming a constant background $B_{y}$ penetration field inside the tail (which is not further explained), an increase in the IMF penetration in the plasma sheet appears to balance the high thermal plasma pressure in this region.

\subsection{The goal of this study}

The main focus of this study lies on the IMF $B_{y}$ and $B_{z}$ dependent large-scale topological structure of the magnetotail and its connection to the shape of the polar cap boundary, as well as on the possible occurrence of polar arcs. Motivated by observations that constant northward IMF with a nonzero IMF $B_{y}$ component leads to a strong twisting of the tail plasma sheet and is a favorable condition for oval-aligned arcs to occur, we investigate, with the help of the GUMICS4 MHD code by Janhunen (1996), how the tail and polar cap boundary respond to different IMF clock angles. The response of the model magnetosphere on an IMF $B_{y}$ sign change is studied to find out about the possible appearance of a moving polar arc (as expected from observations) and the possible rotation of the tail twist (as assumed in the Kullen (2000) polar arc model).

\section{Results}

\subsection{The MHD simulations}

The GUMICS-4 code is a global magnetohydrodynamic model of the magnetosphere. The solar wind and the magnetosphere regions are treated with the ideal MHD equations. The ionosphere is described with electrostatic equations. The model needs as input parameters the solar wind speed, density and pressure, IMF $B_{y}$, IMF $B_{z}$ and the Earth dipole tilt. All runs start with a dipole in a uniform low-density plasma. The solar wind enters the dayside front of the simulation box at the first minute of the run. The simulation box is $32 R_{E}$ long on the dayside and stretches to $224 R_{E}$ on 
Table 1. The GUMICS-4 simulation runs.

\begin{tabular}{lcccccc}
\hline MHD run & $\begin{array}{c}\text { IMF } B_{y} \\
(\mathrm{nT})\end{array}$ & $\begin{array}{c}\text { IMF } B_{z} \\
(\mathrm{nT})\end{array}$ & $\begin{array}{c}\text { IMF clock angle } \\
(\mathrm{deg})\end{array}$ & $\begin{array}{c}\text { Duration of } \\
\text { MHD run }\end{array}$ & $\begin{array}{c}\text { Start of } \\
B_{y} \text { change }\end{array}$ & $\begin{array}{c}\text { Duration of } \\
B_{y} \text { change }\end{array}$ \\
\hline $1 \mathrm{a}$ & -5 & -1 & $100^{\circ}$ & $2 \mathrm{~h}$ & - & - \\
$1 \mathrm{~b}$ & -5 & +1 & $80^{\circ}$ & $2 \mathrm{~h}$ & - & - \\
$1 \mathrm{c}$ & -3.6 & +3.6 & $45^{\circ}$ & $2 \mathrm{~h}$ & - & - \\
$1 \mathrm{~d}$ & -1 & +5 & $10^{\circ}$ & $2 \mathrm{~h}$ & - & - \\
$2 \mathrm{a}$ & $-5 \rightarrow+5$ & +5 & $45^{\circ} \rightarrow 0^{\circ} \rightarrow 45^{\circ}$ & $4 \mathrm{~h}$ & $2: 00 \mathrm{~h}$ & $2 \mathrm{~min}$ \\
$2 \mathrm{~b}$ & $-5 \rightarrow+5$ & +5 & $45^{\circ} \rightarrow 0^{\circ} \rightarrow 45^{\circ}$ & $5 \mathrm{~h}$ & $2: 00 \mathrm{~h}$ & $60 \mathrm{~min}$ \\
\hline
\end{tabular}

the tailward side. In dawn-duskward and north-southward direction the box is $128 R_{E}$ wide. The shell at a distance of $3.7 R_{E}$ from the Earth is defined as the boundary between the magnetosphere and the ionosphere. The boundary conditions at this shell are updated with a new potential distribution every $4 \mathrm{~s}$ by solving the ionospheric equations. Magnetic field lines map from the shell to the high-latitude ionosphere above 60 degrees latitude. The model ionosphere is assumed to be planar and is discretized into a 2-D triangular grid, each grid point of which is further subdivided into 20 nonuniform height levels for the conductivity computation. In the magnetosphere all physical quantities are calculated with time steps of less than a second for a mesh containing about 130000 grid cells. The time steps and the grid size are not constant throughout the simulation box. The code uses subcycles in areas where the time step is refined (obeying the CFL condition) and a semi-automatic grid-refinement is used which depends on the gradients of the physical quantities. In the far tail magnetosphere mesh size and time steps have typical values of $2-4 R_{E}$ and $1 \mathrm{~s}$, respectively, while at the magnetospheric boundaries and in the near-Earth magnetosphere grid spacing and time steps are much reduced. At the boundary shell between ionosphere and magnetosphere, time steps of $0.01-0.03 \mathrm{~s}$ and a grid spacing of $0.5 R_{E}$ are used. The ionospheric grid is fixed in time with about $200 \mathrm{~km}$ between the grid points, although refined to $100 \mathrm{~km}$ in the auroral oval region. The ionospheric grid somewhat oversamples the magnetospheric one since the effective ionospheric resolution provided by the magnetospheric grid is about $300 \mathrm{~km}$. As for this study only large-scale features are of interest, the physical quantities are examined in time intervals of $5 \mathrm{~min}$ and spatial distances of $1 R_{E}$ only. The results presented here are accurate only within these limits. Further details of the MHD code can be found in Janhunen (1996), the ionospheric model is described in Janhunen and Huuskonen (1993), a critical discussion of the MHD model advantages and limitations are described by Janhunen and Palmroth (2001).

Table 1 lists the different MHD runs that are examined in this study. It gives the IMF input value, the IMF clock angle and the duration of the different simulation runs. The IMF clock angle $\theta$ is defined here as the angle between the IMF vector in the $y z$-plane and the positive $z$-axis, ranging from $0^{\circ}$ for pure northward to $180^{\circ}$ for pure southward IMF. In all runs a solar wind speed of $400 \mathrm{~km} / \mathrm{s}$, a solar wind density of $7.3 \mathrm{~cm}^{-3}$ and a solar wind dynamic pressure of $2 \mathrm{nPa}$ have been chosen. The Earth dipole tilt is set to zero to avoid a warping of the tail plasma sheet.

To examine the steady-state magnetosphere during constant IMF with nonzero IMF $B_{y}$ runs $1 \mathrm{a}-1 \mathrm{~d}$ have been performed. The runs are done for IMF clock angles between 100 and 10 degrees. Runs with clock angles higher than 100 degrees are not included, as substorms may occur (which is not the subject of our study). As the highest IMF clock angle input in our simulations is $\theta=100^{\circ}$, the expressions "large clock angle" and "small clock angle" are used further on to describe IMF directions with $\left|B_{y}\right|>B_{z}$ and $\left|B_{y}\right|<B_{z}$, respectively. For a constant solar wind input, as in run $1 \mathrm{a}-1 \mathrm{~d}$, the model magnetosphere reaches an equilibrium state after approximately $1: 30 \mathrm{~h}$. The runs are continued for additionally 30 min to rule out any influence of a transitional change on the magnetospheric topology. All plots shown of the constant IMF runs $1 \mathrm{a}-1 \mathrm{~d}$ give the state of the magnetosphere $2 \mathrm{~h}$ after the startup.

To study a sign change of IMF $B_{y}$, the MHD runs $2 \mathrm{a}$ and $2 \mathrm{~b}$ have been done. The IMF input parameters consist of constant positive IMF $B_{z}$ during the entire time and IMF $B_{y}$ negative before and positive after an IMF $B_{y}$ sign change which starts after two hours constant IMF at the dayside boundary of the simulation box. In each case, the IMF is kept constant for an additional two hours following the sign change. The difference between run $2 \mathrm{a}$ and $2 \mathrm{~b}$ is the speed of the IMF $B_{y}$ sign change. In the first case, IMF $B_{y}$ changes sign within two minutes, in the second case a slow, linear change within one hour is enforced.

\subsection{MHD results for constant IMF}

Figure 1 presents the state of the ionosphere and magnetotail for the simulation runs after two hours constant solar wind input. The columns (from left to right) show the simulation results for run $1 \mathrm{a}, 1 \mathrm{~b}, 1 \mathrm{c}$ and $1 \mathrm{~d}$. In the first row the northern ionosphere is shown with the open/closed field line boundary (light blue dotted lines) overlaid on a plot showing the upward (blue) and downward (red) field-aligned current regions. The second to last rows consist of plasma pressure isocontour plots (in arbitrary units) for tail cross sections at $x=-10, x=-30, x=-60$ and $x=-120 R_{E}$. The color scale 

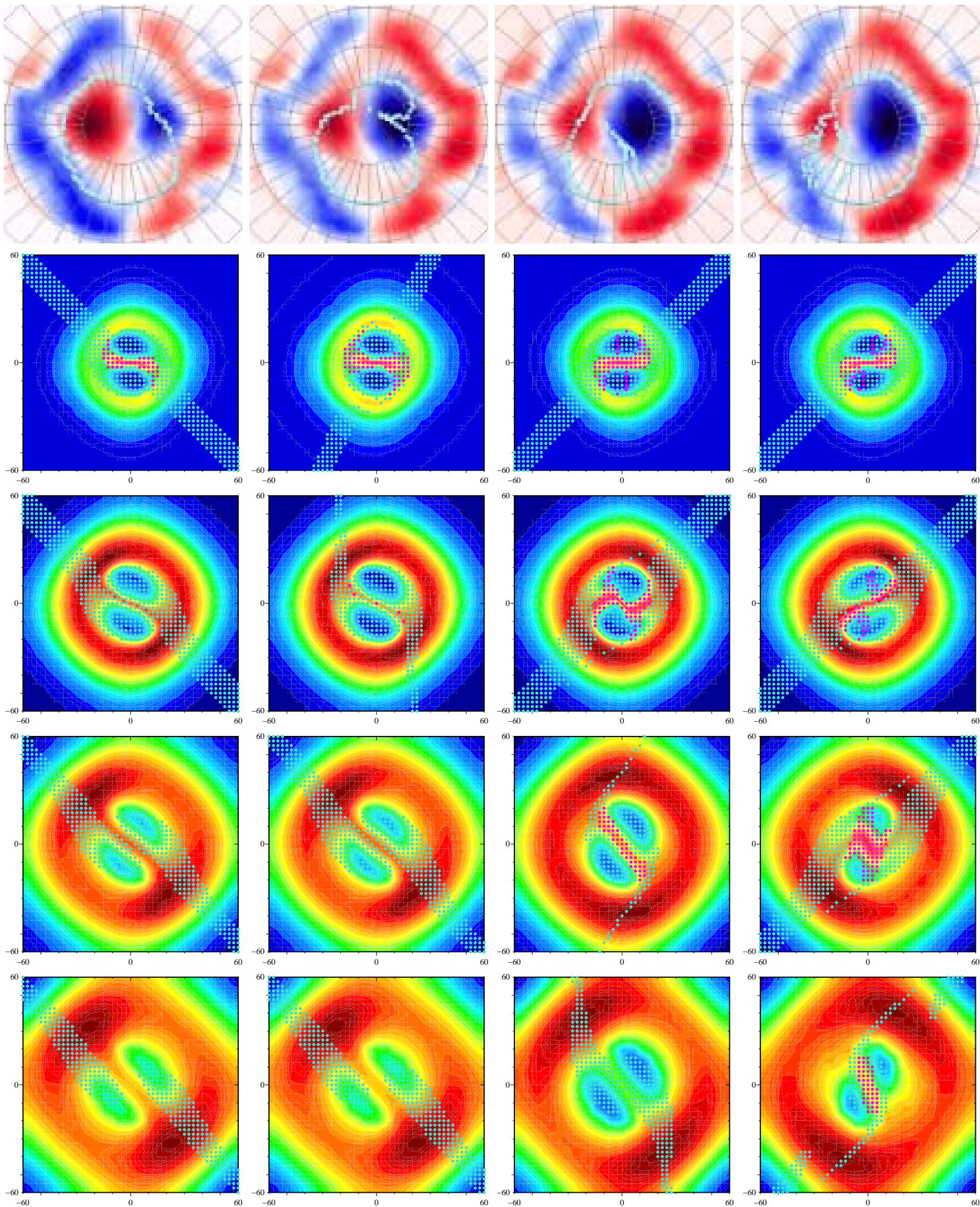

Fig. 1. Simulation results after two hours constant IMF. The columns (from left to right) show the magnetosphere for IMF clock angles of $100^{\circ}, 80^{\circ}, 45^{\circ}$ and $10^{\circ}$ and an IMF magnitude of $5.1 \mathrm{nT}$ (runs 1a, 1b, 1c and 1d). The first row contains plots of the northern ionosphere with the open/closed field line boundary (light blue line) overlaid on the upward (blue) and downward (red) field-aligned currents. The plots give a 2-D view on the polar cap in geomagnetic coordinates with noon pointing to the top. The second to last row show tail cross sections at $x=-10, x=-30, x=-60$ and $x=-120 R_{E}$ (looking downtail in GSM coordinates). In the tail plots the regions of closed (pink dots) and open field lines (light blue dots) are overlaid on plasma pressure isocontour lines (in arbitrary units). 
reaches from blue to red, representing lowest to highest pressure. Overlaid on these plots are the regions of open field lines (blue dots) and closed field lines (pink dots). Figure 2 shows the same type of plots for run $2 \mathrm{a}$, the fast IMF $B_{y}$ sign change case. The columns (from left to right) represent the state of the magnetosphere $6 \mathrm{~min}$ before, $7 \mathrm{~min}$ after, $22 \mathrm{~min}$ after and $37 \mathrm{~min}$ after the center of the IMF $B_{y}$ signchange region $\left(B_{y}=0\right)$ reached the dayside magnetopause at $x=10 R_{E}$. In deviation from Fig. 1 the last row of Fig. 2 shows tail cross sections at $x=-90 R_{E}$. The main results of this Figure are described in the next subsection. Here, the first column of Fig. 2 (run 2a just before the sign change appears) is compared to column 3 of Fig. 1 (run 1c) to find out about a possible difference between strong (7.1 nT) and average IMF (5.1 nT) for the same IMF clock angle of $45^{\circ}$.

While the field-aligned current (FAC) pattern is for all MHD runs nearly unchanged, the polar cap boundary (here defined as the boundary between open and closed field lines) differs much for the different constant IMF cases (run 1a1d). The polar cap is more contracted for small IMF clock angles, and the dawnside polar cap boundary is for all clock angles displaced poleward as expected in the Northern Hemisphere for negative IMF $B_{y}$. The polar cap boundary of the Southern Hemisphere (not shown here) is anti-symmetrically shaped with respect to the noon-midnight meridian, the poleward displacement occurs there on the dusk side of the polar cap.

As expected from the highly contracted polar cap for small clock angles, the closed field line region extends in the magnetotail to much higher latitudes for small IMF clock angles than for large clock angles. The tailward extension of the closed field line region depends on the IMF clock angle as well. Table 2 gives the tailward length of the closed field line region for all constant MHD runs, the MHD runs $1 \mathrm{a}-1 \mathrm{~d}$ and for run 2 a just before the $B_{y}$ sign change took place. Only for small IMF clock angles does the closed field line region extend to the far tail. Comparing the $45^{\circ}$ clock angle cases with weak (run 1c) and strong (run 2a before sign change) IMF magnitude, the closed field line region is slightly shorter in the latter case. The open/closed field line boundary overlaps approximately with the plasma sheet boundary for all but the $10^{\circ}$ IMF clock angle case (run 1d). In the latter case the region of closed field lines extends high into the tail lobes.

The region of open field lines outside the magnetotail is directed along the IMF clock angle axis but with a slight clockwise displacement from it (looking tailward). Inside the near-Earth tail the open field line region surrounds the closed field line region. Far downtail the closed field lines have disappeared and for large IMF clock angles the northern and southern wings of the open field line regions are much displaced from each other in the dawn-dusk direction such that they hardly overlap with the lobes.

As expected for negative IMF $B_{y}$ the plasma sheet (high pressure region inside the tail) is twisted in a clockwise direction around the $x$-axis (looking tailward) for each of the constant IMF runs. Only for small IMF clock angles and/or high IMF magnitude is the plasma sheet twist strong and it
Table 2. The tailward extension of the closed field line region (CFR length) for the constant IMF clock angle runs.

\begin{tabular}{|c|c|c|c|c|c|}
\hline MHD run & 1a & $1 b$ & $1 \mathrm{c}$ & $2 a^{*}$ & $1 d$ \\
\hline IMF Clock angle & ${ }^{1} 100^{\circ}$ & $180^{\circ}$ & $1_{45^{\circ}}$ & $245^{\circ}$ & ${ }^{1} 10^{\circ}$ \\
\hline CFR length & $20 R_{E}$ & $20 R_{E}$ & $34 R_{E}$ & $28 R_{E}$ & $85 R_{E}$ \\
\hline
\end{tabular}

extends over the entire tail cross section. For large IMF clock angles the plasma sheet twists only near the flanks. The twisting increases clearly with the distance from the Earth for the $45^{\circ}$ and $10^{\circ}$ clock angle cases (run 1c and 1d). For the $100^{\circ}$ and $80^{\circ}$ cases (run $1 \mathrm{a}$ and $1 \mathrm{~b}$ ) the plasma sheet is in the center of the far tail twisted in the opposite direction. For all but very small IMF clock angles a typical tail pressure morphology with lobes and plasma sheet occurs even in regions tailward of the last closed field line. The plasma sheet thickness and the dawn-duskward extension of the lobes are dependent on the IMF clock angle as well. In the near-Earth tail the plasma sheet is thick only for small IMF clock angles. In the far tail the dawn-duskward width of the lobes decreases with decreasing IMF clock angles.

Figure 3 shows the $B_{y} B_{z}$ vector components of the additional magnetic field occurring in the constant IMF cases a1 (upper row) and 1d (lower row). The plots are done for tail cross sections at $x=-10 R_{E}$ (left column) and $x=-60 R_{E}$ (right column). To extract the symmetric part of the tail magnetic field, the along the $x z$-plane mirrored $B_{y} B_{z}$ vectors have been subtracted from the original $B_{y} B_{z}$ vector field and then been divided by two. Assuming the tail magnetic field for zero IMF $B_{y}$ is completely symmetric with respect to the $x z$-plane, this gives the additional $B_{y}$ field appearing typically during nonzero IMF $B_{y}$.

For both IMF clock angle cases the IMF is slightly draped around the magnetopause. A large additional $B_{y}$ component, pointing in the same direction as IMF $B_{y}$, occurs inside the magnetosphere. In the near-Earth tail the $B_{y}$ extra field is strong near the equatorial plane and weak at high latitudes (at $z=0 R_{E}$, the extra $B_{y}$ field has approximately the same or even slightly higher values than the IMF $B_{y}$ component). This structure is preserved to around $x=-45 R_{E}$. Further downtail, the extra $B_{y}$ field weakens and is more uniformly distributed. At the tailward end of the simulation box (not shown here) the extra $B_{y}$ field is weaker than IMF $B_{y}$ for all IMF clock angle runs.

Figure 4 gives an equatorial view on the magnetosphere for MHD run 1a (first plot) and 1d (second plot). The $B_{x} B_{y}$ vector components are plotted down to a distance of $4 R_{E}$ from the Earth. In case IMF $B_{y}=0$, the magnetic field in the equatorial plane of the magnetosphere is expected to have only a z-component. Hence, the $B_{x} B_{y}$ plots show the additional $B_{x} B_{y}$ field appearing in the equatorial plane of the tail during nonzero IMF $B_{y}$. Figure 5 contains the same type of plots for the sudden IMF $B_{y}$ sign change (run $2 \mathrm{a}$ ). 

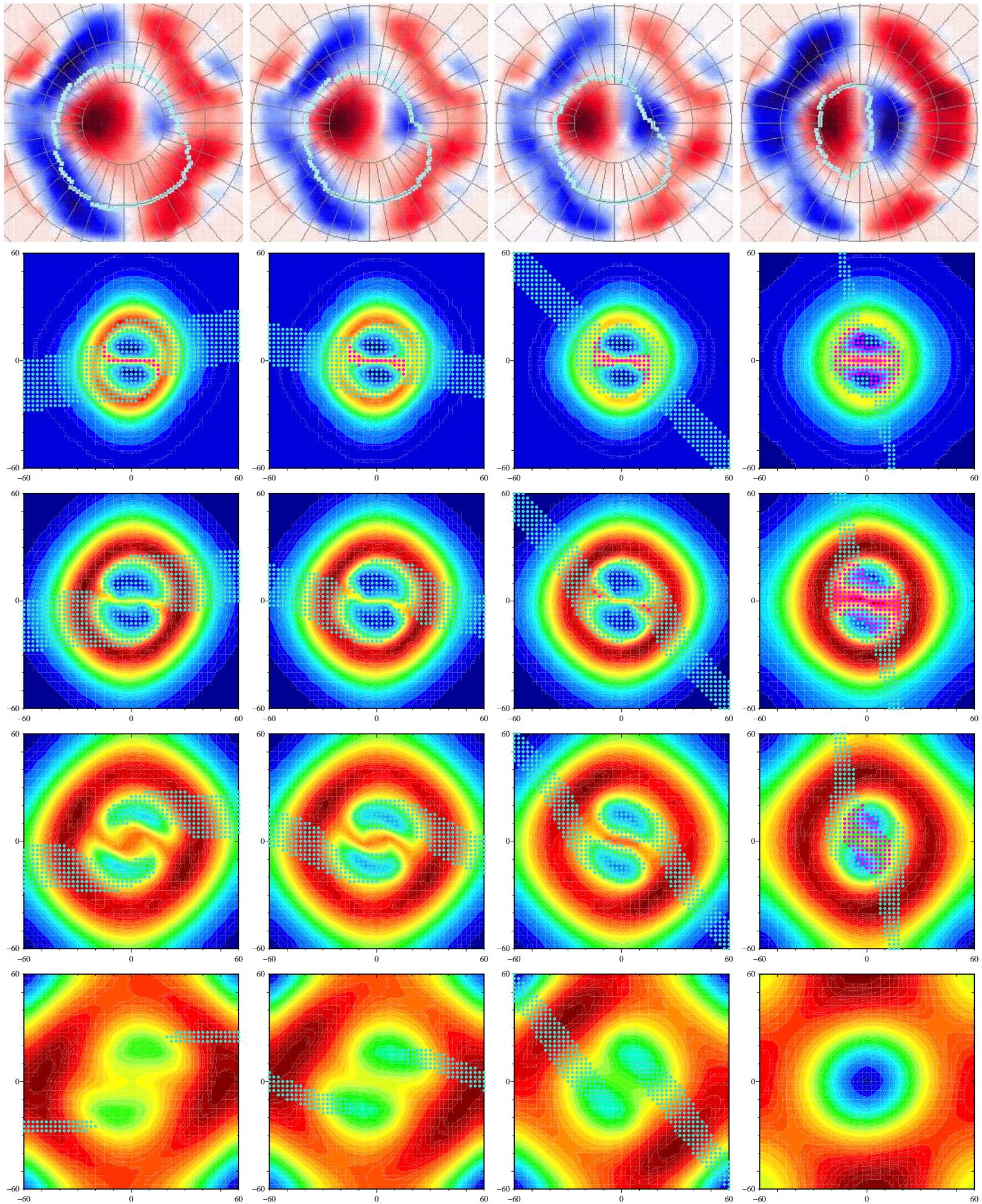

Fig. 2. Simulation results for an MHD run with positive IMF $B_{z}$ of $+5 \mathrm{nT}$ and a fast ( $\left.2 \mathrm{~min}\right)$ IMF $B_{y}$ sign change from $-5 \mathrm{nT}$ to $+5 \mathrm{nT}$ after two hours constant IMF (run 2a). The columns (from left to right) show the state of the magnetosphere $-8,7,22$ and 37 min after the center of the IMF $B_{y}$ sign-change region $\left(B_{y}=0\right)$ has passed the dayside magnetopause (at $x=10 R_{E}$ ). The first row shows the northern ionosphere. The second to last row show tail cross sections at $x=-10, x=-30, x=-60$ and $x=-90 R_{E}$. The plots are defined in the same way as in Fig. 1. 

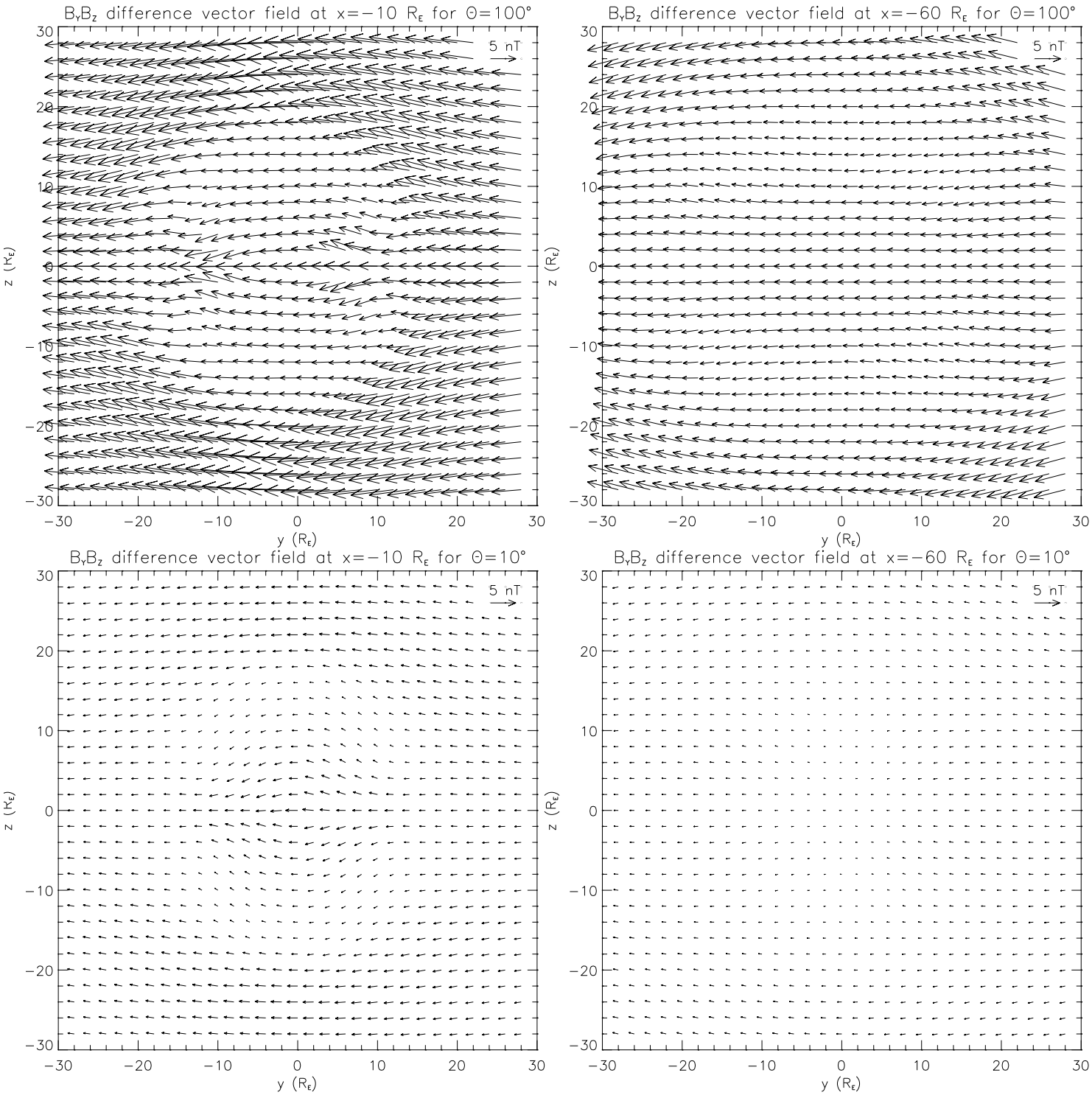

Fig. 3. The additional magnetic field that occurs in the tail during nonzero IMF $B_{y}$. In the Figure, two different tail cross sections for the constant IMF runs $1 \mathrm{a}$ (up) and $1 \mathrm{~d}$ (down) are shown. The plots are produced by mirroring the $B_{y} B_{z}$ vector components of the corresponding tail cross section along the $z$-axis, subtracting the mirror vectors from the original vectors and dividing the results by two.

It shows the $B_{x} B_{y}$ vector component plots 6 min before (top), 7 min after (middle) and 22 min after (bottom) the IMF $B_{y}$ sign reversal reached the dayside magnetopause. The main results for the IMF $B_{y}$ sign-change case are described in the next subsection. Here, only the first plot is addressed, corresponding to a constant $45^{\circ} \mathrm{IMF}$ run for $7.1 \mathrm{nT}$. In the format shown here, its deviation to the results of run $1 \mathrm{~d}$ is negligible such that it illustrates even the results of the weak $(5.1 \mathrm{nT})$ $45^{\circ}$ case fairly well.

The $B_{x} B_{y}$ vector plots for the different IMF clock angles have several features in common. The IMF $B_{y}$ field is strongly compressed near the dayside magnetopause and drapes around the magnetosphere. In the region closest to the Earth which is dominated by the Earth dipole field $\left(|x|<8 R_{E}\right)$ a nearly uniform extra $B_{y}$ component is found having approximately the same magnitude as the IMF itself. Tailward of this region, the $B_{x} B_{y}$ vector components have a sunward draping pattern centered around the $x$-axis. The draping pattern reduces tailward such that in the very far tail the $B_{x} B_{y}$ component lines are directed in the dusk-dawn direction. The draping inside the tail reflects the twisting of the plasma sheet seen in Fig. 1, first and last column, and Fig. 2, first column. A positive (negative) component of $B_{x}$ in the equatorial plane causes a southward (northward) displacement of the current sheet (surface containing the turning points of the closed field lines) from the equatorial plane. The stronger draping for the small IMF clock angle case (run 1d) is connected to a stronger and more uniform twist around the $x$-axis, the tailward draping downtail of $25 R_{E}$ near the $x$-axis for the $100^{\circ}$ IMF clock angle case (run 1a) 

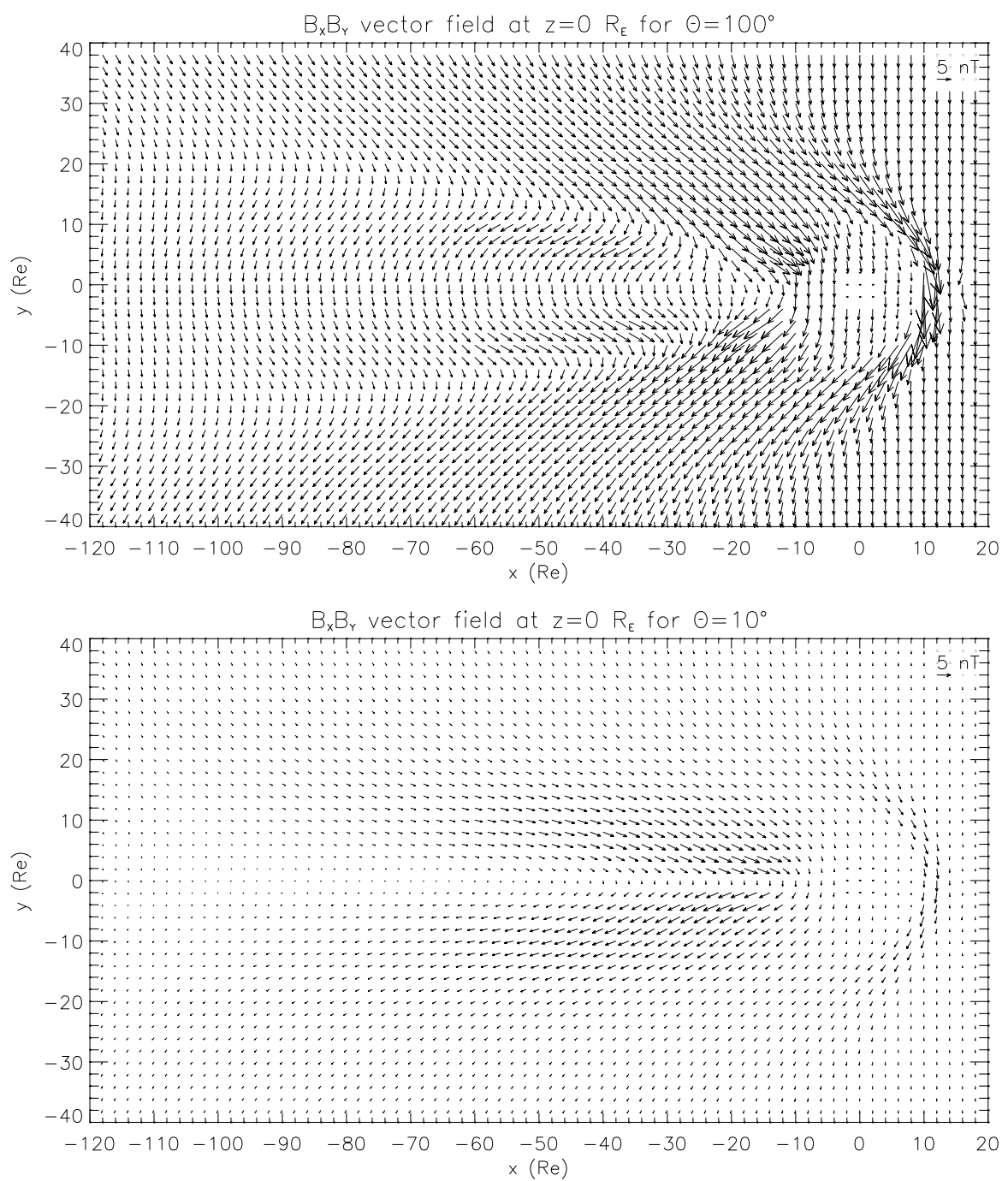

Fig. 4. The magnetic field in the equatorial plane for two constant IMF runs $1 \mathrm{a}$ (up) and $1 \mathrm{~d}$ (down). The vectors are plotted every $2 R_{E}$ down to a distance of $4 R_{E}$ from the Earth is center.

corresponds to the oppositely twisted plasma sheet in the far tail center (Fig. 1, column 1, row 4).

\subsection{MHD results for an IMF $B_{y}$ sign change}

As mentioned above, Fig. 2 illustrates the change in the polar cap and the tail for the fast IMF $B_{y}$ sign change case (run 2a). The plots show the state of the magnetosphere when the IMF $B_{y}$ sign reversal starts at the dayside boundary of the simulation box, and 5, 20 and $35 \mathrm{~min}$ after the first significant change of the polar cap boundary is seen. The ionosphere plots in the first row of Fig. 2 show that the polar cap boundary changes drastically after an IMF $B_{y}$ sign change. A bridge of closed field lines starts to develop at the dawn/noon edge of the polar cap boundary and moves within $50 \mathrm{~min}$ from dawn to dusk over the entire polar cap of the Northern Hemisphere. In the Southern Hemisphere (not shown here) an identical bridge starts to appear on the dusk side and then moves in the opposite direction until the dawn side polar cap boundary is reached. The bridge of closed field lines inside the northern (southern) polar cap is connected to a finger of closed field lines in the tail bifurcating the northern (southern) tail lobe. The fingers of closed field lines in the tail move into the same direction as the bridge of closed field lines in the polar cap of the corresponding hemisphere.

Figure 6 shows the tailward extension of the closed field line region and the location of the IMF $B_{y}$ sign reversal regions versus time for run $2 \mathrm{a}$ (thin line and dark grey region) and run $2 \mathrm{~b}$ (thick line and light grey region). The time axis is given in minutes after the center of the IMF $B_{y}$ sign-change region has not passed the dayside magnetopause. The time intervals during which a bifurcation of the polar cap occurs are marked with dotted lines. As can be seen from Fig. 6 the length of the closed field line region varies greatly during the tailward propagation of an IMF $B_{y}$ sign reversal. As long as the center of the IMF $B_{y}$ sign-change region, has not passed the tailward end of the closed field line region, the 

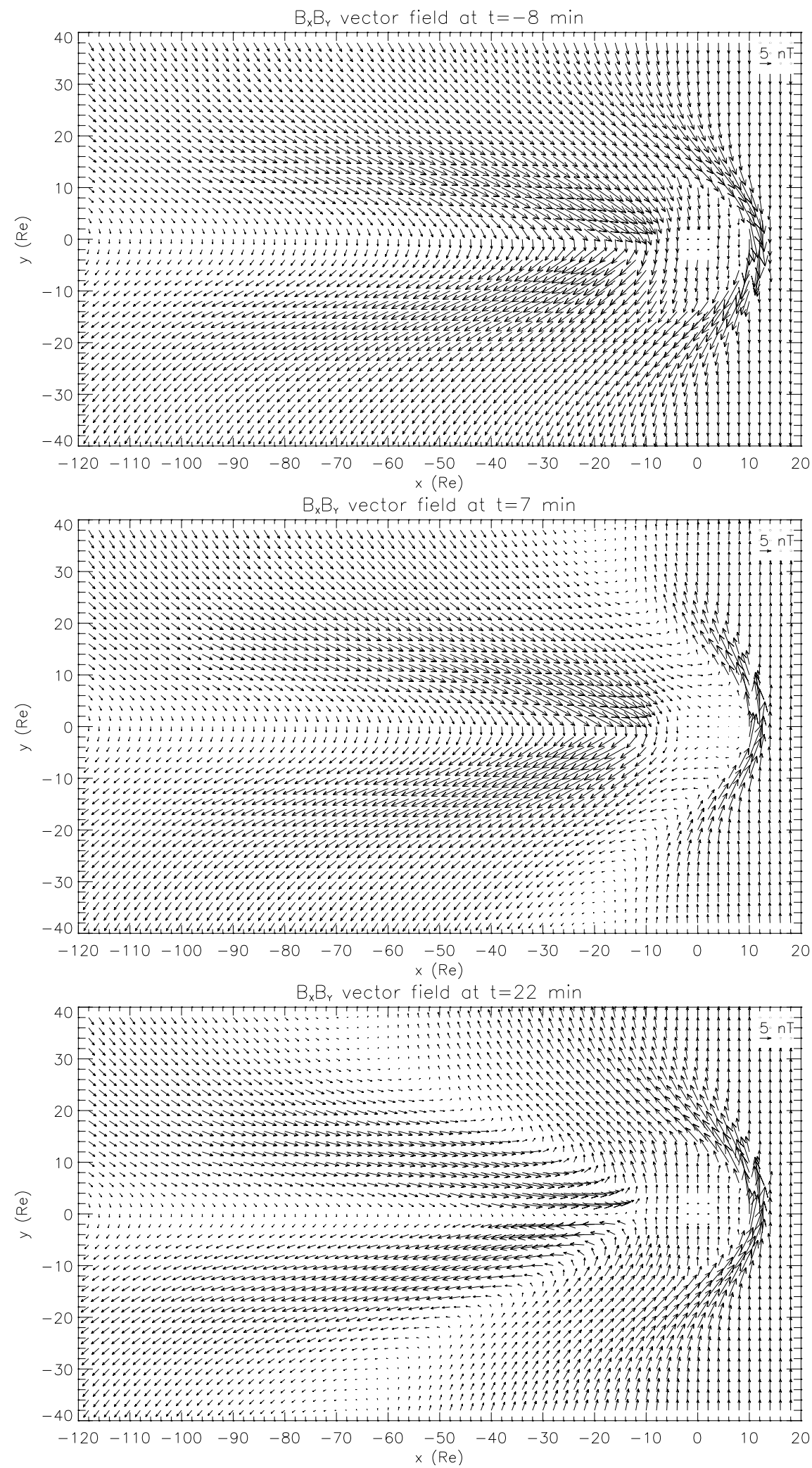

Fig. 5. The magnetic field in the equatorial plane for different points of time during the fast IMF $B_{y}$ sign-change case (run $2 a$ ). 


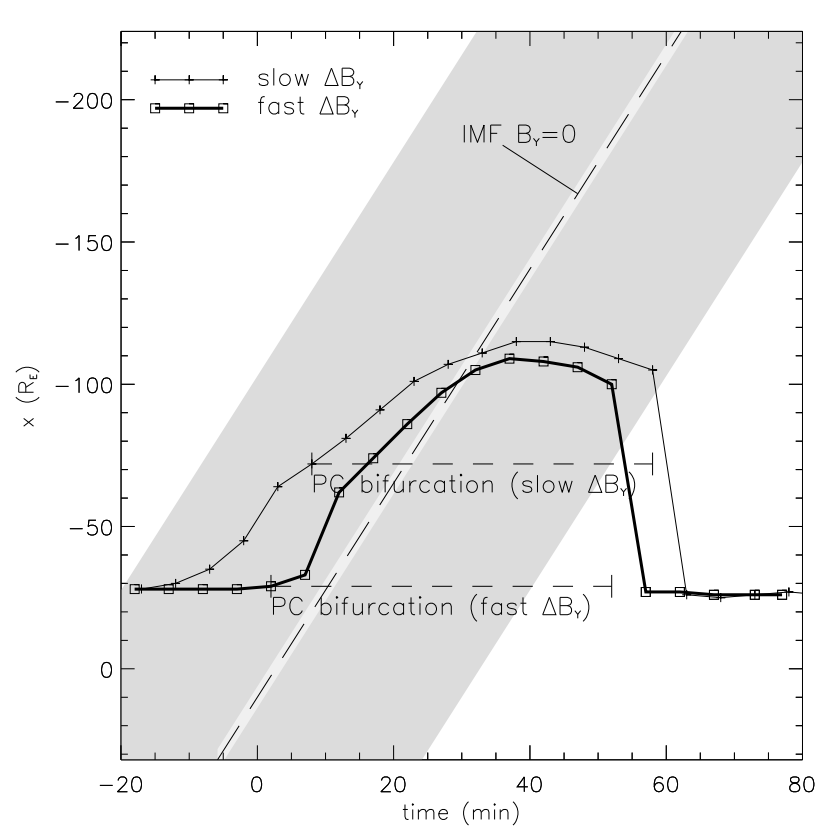

Fig. 6. The length of the closed field line region versus time for the fast (run 2a) and the slow (run 2b) IMF $B_{y}$ sign-change case. The time is given in minutes after the center of the sign-change region has passed the dayside magnetopause. The tailward propagating IMF sign-change region and the time interval during which a polar cap bifurcation occurs are marked for both runs.

closed field line region grows rapidly beyond $x=-100 R_{E}$ in both IMF $B_{y}$ sign-change cases. The closed field line region remains extremely stretched for another $25 \mathrm{~min}$ until it suddenly returns to its original length. The polar cap bifurcation starts in both sign-change runs some minutes after the center of the IMF $B_{y}$ sign reversal has passed the dayside magnetopause and ends when the far tail part of the closed field line region disappears and the tail returns to its original length.

The process that leads to the return of a ground state for constant duskward IMF $B_{y}$ with a short closed field line region is rather complicated. This is illustrated in Fig. 7 and Fig. 8. In Fig. 7 the open (grey) and closed field line regions (black) of run 2a are shown in the $x y$-plane at $z=0 R_{E}$ (left column) and $z=15 R_{E}$ (right column) for different points of time after the IMF $B_{y}$ sign-change region center has passed the dayside magnetopause. The first four time points correspond to the time points shown in Figs. 1 and 2. The last two time points are chosen just before and just after the polar cap bridge disappears. Figure 8 shows the magnetic field line topology in 3-D for the same run and same time points as in Fig. 7.

The left column of Fig. 7 shows that, when the tail has reached its maximum length (at $22 \mathrm{~min}$ ), the tailward part of the closed field line region becomes separated from the earthward part in the equatorial plane. The far tail is connected to the northern (southern) ionosphere only by a thin, tail-aligned bridge of closed field lines moving from dawn to
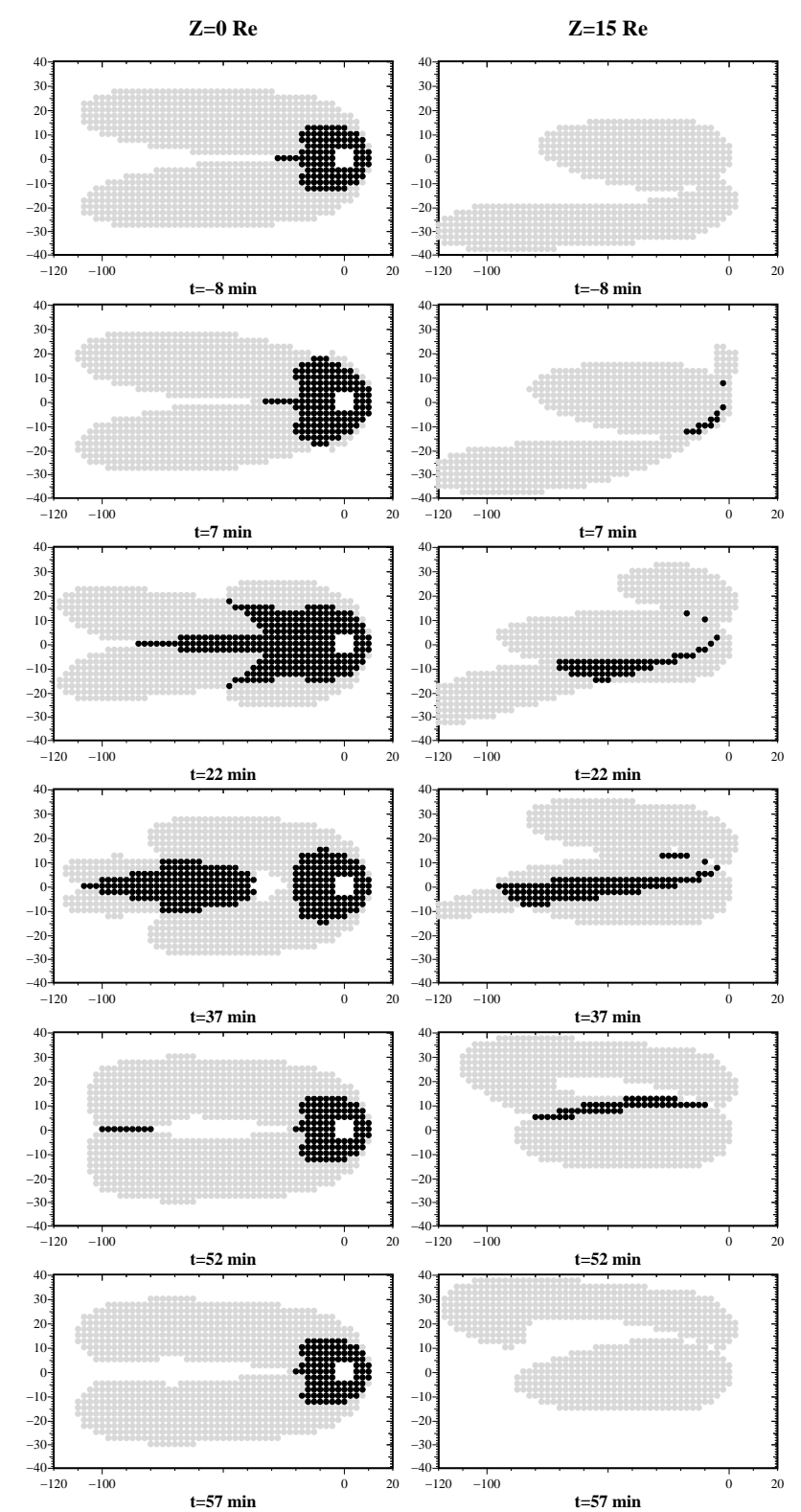

Fig. 7. Open (grey) and closed (black) field line region in the equatorial plane (left column) and above it (right column) for different points of time during the fast IMF $B_{y}$ sign-change run (run 2a).

dusk (dusk to dawn) in the northern (southern) high-latitude lobe (Fig. 7, right column). This bridge of far tail closed field lines maps to the model polar arcs (not shown here). The tailward closed field line part reduces in length and width until it disappears, together with the closed field line bifurcation in the ionosphere and the tail. The disappearance is not enforced by the limited length of the simulation box. The IMF $B_{y}$ sign reversal is at that time point still located earthward of the nightside simulation box boundary (see Fig. 6, run 2a). It is difficult to define reconnection in an MHD simulation. However, studying the field line topology in detail, the location of the separation region between open, closed and solar wind field lines can be found. Before the IMF $B_{y}$ 

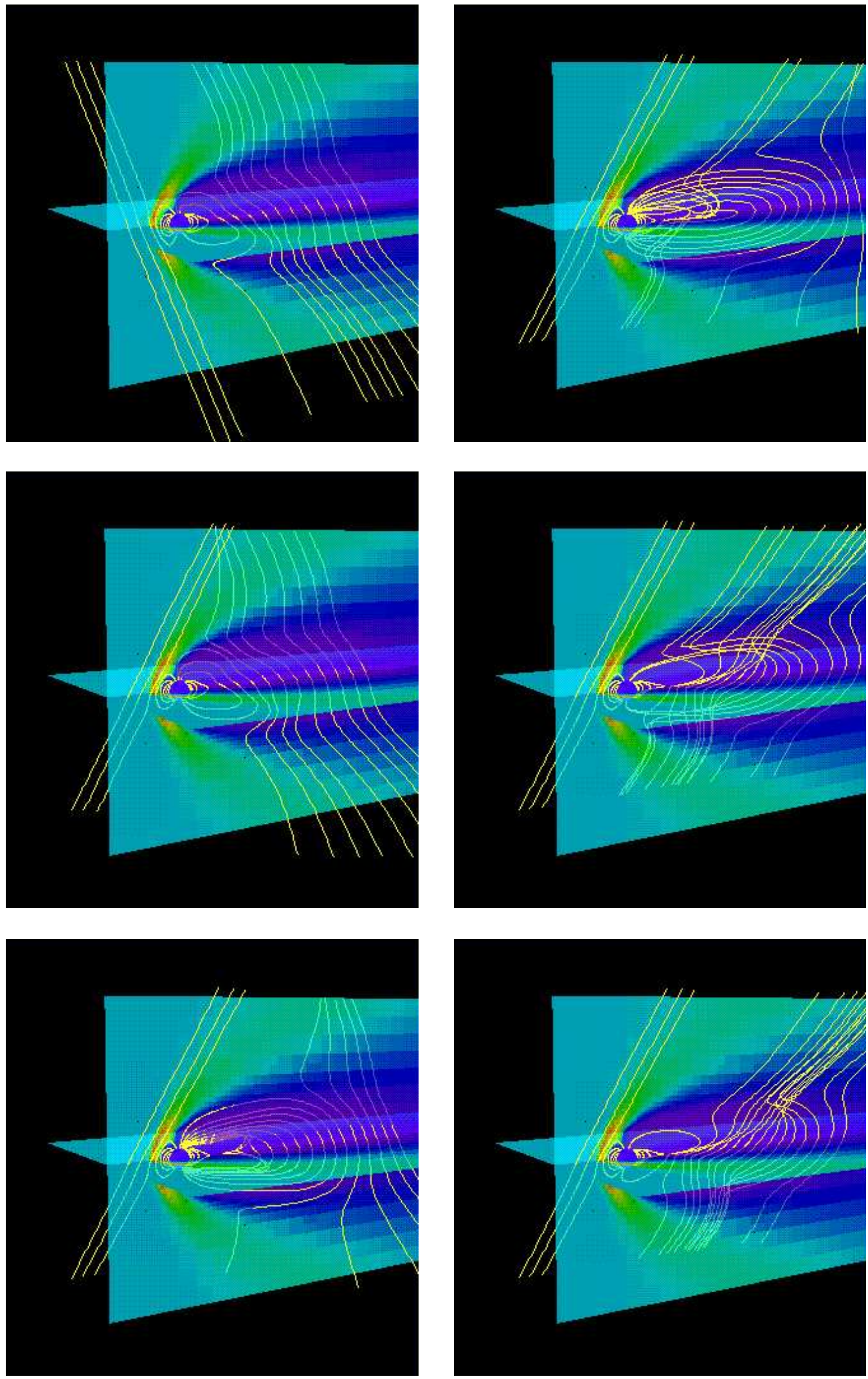

Fig. 8. A 3-D view on field lines crossing the $x$-axis for the fast IMF $B_{y}$ sign change case (run $2 a$ ). The plots show the state of the magnetosphere $-8,7,22 \mathrm{~min}$ (first column from top to bottom) and 37, 52, $57 \mathrm{~min}$ (second column, from top to bottom) after the center of the IMF $B_{y}$ sign-change region $\left(B_{y}=0\right)$ has passed the dayside magnetopause. 
sign change region has reached the magnetosphere (Fig. 8, first plot), the separation region in the Northern (Southern) Hemisphere is located at the high-latitude lobe, poleward of the cusps and displaced toward dawn (dusk), as expected for dawnward IMF with a northward $B_{z}$ component. Immediately after the IMF $B_{y}$ sign change region reached the magnetosphere, the reconnection region moves from the dawn to the dusk lobe in the Northern Hemisphere and in opposite direction in the Southern Hemisphere lobe. New regions of open field lines appear on the dawn (dusk) flanks of the northern (southern) tail. While the closed field line region extends further and further tailward, the new regions of open field lines increase and the old tailward regions of open field lines decrease. Interestingly, both the new and the old open field lines reconnect with the solar wind field on the northern dusk (southern dawn) high-latitude lobes. When the closed field line region has reached its maximum length, the field line topology in the mid-tail becomes extremely complicated (Fig. 8, right column). Solar wind field lines occur now in the mid-tail near the $x$-axis, separated from the near-Earth and far-tail closed field line regions by open field lines. The open field lines earthward and tailward of the solar wind field lines in the mid-tail, as well as the old open field lines tailward of the last closed field line, are all associated with the same high-latitude lobe reconnection region. The region of solar wind field lines in the mid-tail increases at the expense of the decreasing tailward closed field line region. After the last closed field line has disappeared, the tail turns into a steadystate case for duskward IMF $B_{y}$. The last plot of Fig. 8 shows the field line distribution just after the last closed field line has disappeared in the far tail, where the tailward solar wind field lines are still strongly deformed.

In Fig. $9 B_{y} B_{z}$-difference vector plots are shown for the fast IMF $B_{y}$ sign-change case (run $2 \mathrm{a}$ ) in tail cross sections at $x=-10,-30,-60$ and $-120 R_{E} 22 \mathrm{~min}$ after the IMF $B_{y}$ sign-change region has passed the dayside magnetopause. The difference vector plots are done in the same way as those of Fig. 3, showing the $B_{y} B_{z}$ vector components of the additional field inside the tail. As can be seen from Fig. 5 and Fig. 9 the location of the sign-change center $\left(B_{y}=0\right)$ is situated like a sunward pointing cone around the $x$-axis. Outside the cone, the extra magnetic field points toward dusk, in the direction of positive IMF $B_{y}$. Inside the cone, the extra $B_{y}$ field is still negative and has a distribution similar to the $B_{y}$ extra field of the constant IMF $B_{y}<0$ cases $1 \mathrm{a}-1 \mathrm{~d}$ (see Fig. 3). The additional $B_{y}$ field is stronger near the plasma sheet than in the lobes. The nonuniformity of the extra field inside the tail decreases with the distance from the Earth.

The $B_{x} B_{y}$-vector component plots in Fig. 5 illustrate the propagation of the IMF $B_{y}$ sign reversal in the equatorial plane. The cone of zero $B_{y}$ moves tailward with time and becomes more and more narrow the further downtail it propagates. This indicates that outside the tail the sign reversal moves tailward with solar wind speed, and toward the tail center the effect of the IMF $B_{y}$ sign reversal is more and more delayed. Comparing the last two $B_{x} B_{y}$-vector plots with the first plot of Fig. 5, it can be seen that the location of the sign-change region center $\left(B_{y}=0\right)$ in the equatorial plane is quite similar to the draping pattern of the $B_{x} B_{y}$-vector component lines in the constant IMF $B_{y}$ case.

Important results are found from comparing the shape of the plasma sheet with the shape of the closed field line region in Fig. 2. In contrary to the closed field line region the plasma sheet does not bifurcate the lobes during the tailward propagation of the IMF $B_{y}$ sign change reversal. It changes its IMF $B_{y}$ induced twist first in the near Earth and then in the far tail such that in an intermediate state the near-Earth and far-tail regions are oppositely twisted. The plasma sheet resembles at times a horizontal " $\mathrm{S}$ " as the twist reversal in one tail cross section starts at the flanks and then propagates to the tail center. In one tail cross section it takes (dependent on the distance from the Earth) 15 to 45 min until the twist rotation is completed. The bifurcation of the closed field line region disappears as soon as the entire tail plasma sheet has changed its twist.

In Fig. 10 a few plots of the slow IMF $B_{y}$ sign change case (run 2b) are shown. The first row shows the northern polar cap boundary and the second row shows the tail cross sections at $x=-30 R_{E}, 13 \mathrm{~min}$ (left column), $28 \mathrm{~min}$ (middle column) and $43 \mathrm{~min}$ (right column) after the center of the IMF $B_{y}$ sign reversal ( $\left.B_{y}=0\right)$ reached the dayside magnetopause. The time points are chosen such that the first (second, third) column gives the state of the magnetosphere five minutes ( $20 \mathrm{~min}, 35 \mathrm{~min}$ ) after the start time of the polar cap bifurcation. This makes the plots comparable to those of Fig. 2.

The main difference between the fast (run 2a) and slow (run 2b) IMF $B_{y}$ sign-change runs are the width of the closed field line bridge in the polar cap and the width of the closed field line fingers bifurcating the tail. The location of the bifurcation, the lifetime of the dawn-duskward moving bridge and the topological change of the tail plasma sheet are qualitatively the same for the slow and for the fast IMF $B_{y}$ signchange runs. Even in run $2 \mathrm{~b}$ the plasma sheet starts to change its twist in the near-Earth tail and at the flanks; it does not bifurcate such that the plasma sheet boundary does not overlap with the bifurcated closed field line region in the tail. The tailward increase and decrease of the closed field line region develops qualitatively in the same way as in run $2 a$. Even in run $2 b$ the near-Earth tail is separated from the far-tail region in the equatorial plane and during the last $15 \mathrm{~min}$ a bifurcation of the closed field line region in tail and ionosphere is seen. However, as shown in Fig. 6, the tail grows faster and stretches further tailward in the slow IMF $B_{y}$ sign change run than in the fast sign change run.

\section{Discussion}

3.1 The constant IMF clock angle cases: comparison with earlier results

The general topological changes inside the model magnetosphere induced by constant IMF $B_{y}$ are confirmed by 

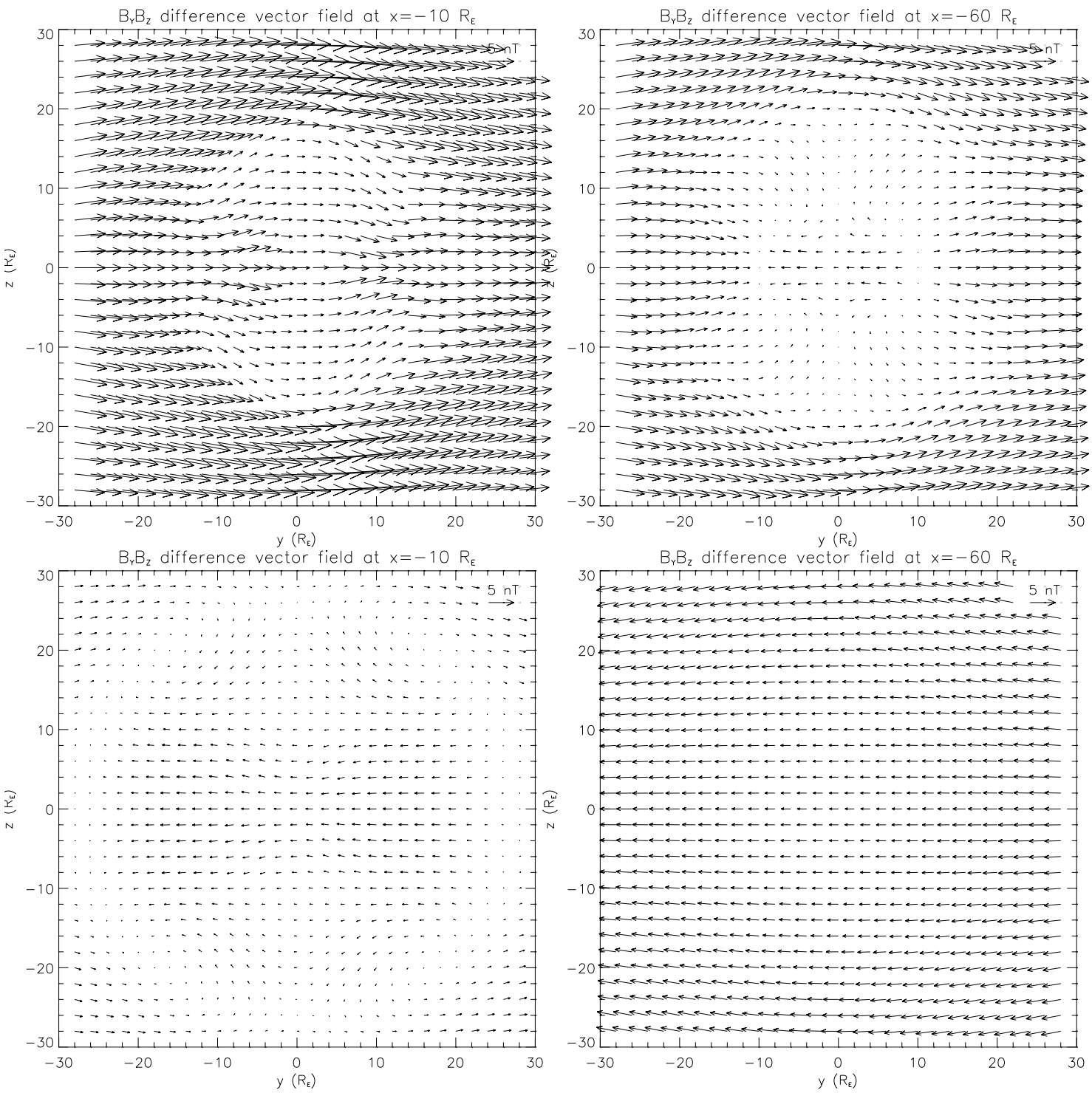

Fig. 9. The additional magnetic field shown at different tail cross sections during the fast IMF $B_{y}$ sign-change case (run 2a). The plots are produced in the same way as Fig. 3 and show the additional magnetic field 22 min after the center of the sign-change region has passed the dayside magnetopause.

observations. This includes the occurrence of an additional $B_{y}$ field in the tail (often referred to as "IMF $B_{y}$ penetration field") having the same direction as IMF $B_{y}$ with a maximum value along the neutral sheet (Lui, 1986; Borovsky et al., 1998), the twisting of the tail plasma sheet with the strongest twist near the flanks (Kaymaz et al., 1994), the tailward increase of the twisting (Tsyganenko, 1998) the small displacement of the open field line region outside the tail from the IMF direction (Kaymaz and Siscoe, 1998), and the dawn-dusk displacement of the open/closed field line boundary in the ionosphere (Cowley, 1981). These IMF $B_{y}$ effects have been reported from MHD studies as well (Kaymaz et al., 1995; Brecht, 1981; Fedder and Lyon, 1995). The preservation of the magnetospheric plasma structure tailward of the last closed field line has been seen in the simulations by Song et al. (1999). Some of the IMF clock angle dependent changes in the magnetospheric topology that are found in our simulations have been indicated in data studies. The increase of the tail twisting and the decrease of the polar cap for small clock angles have been described in the statistical studies by Owen et al. (1995) and Makita et al. (1988), respectively. Statistical results of a concave form of the plasma sheet in the far tail, (Owen et al., 1995), may be an indication for the oppositely twisted far-tail center occurring for IMF clock angles around $90^{\circ}$ in our simulations, as usually solar wind conditions are such that $\left|\operatorname{IMF} B_{y}\right|>\left|\operatorname{IMF} B_{z}\right|$. Other IMF clock angle effects are difficult to observe, they are so far only seen in MHD simulations, such as a long closed field line region for small IMF clock angles (Walker et al., 1999) or small IMF magnitude (Song et al., 1999), the IMF clock 

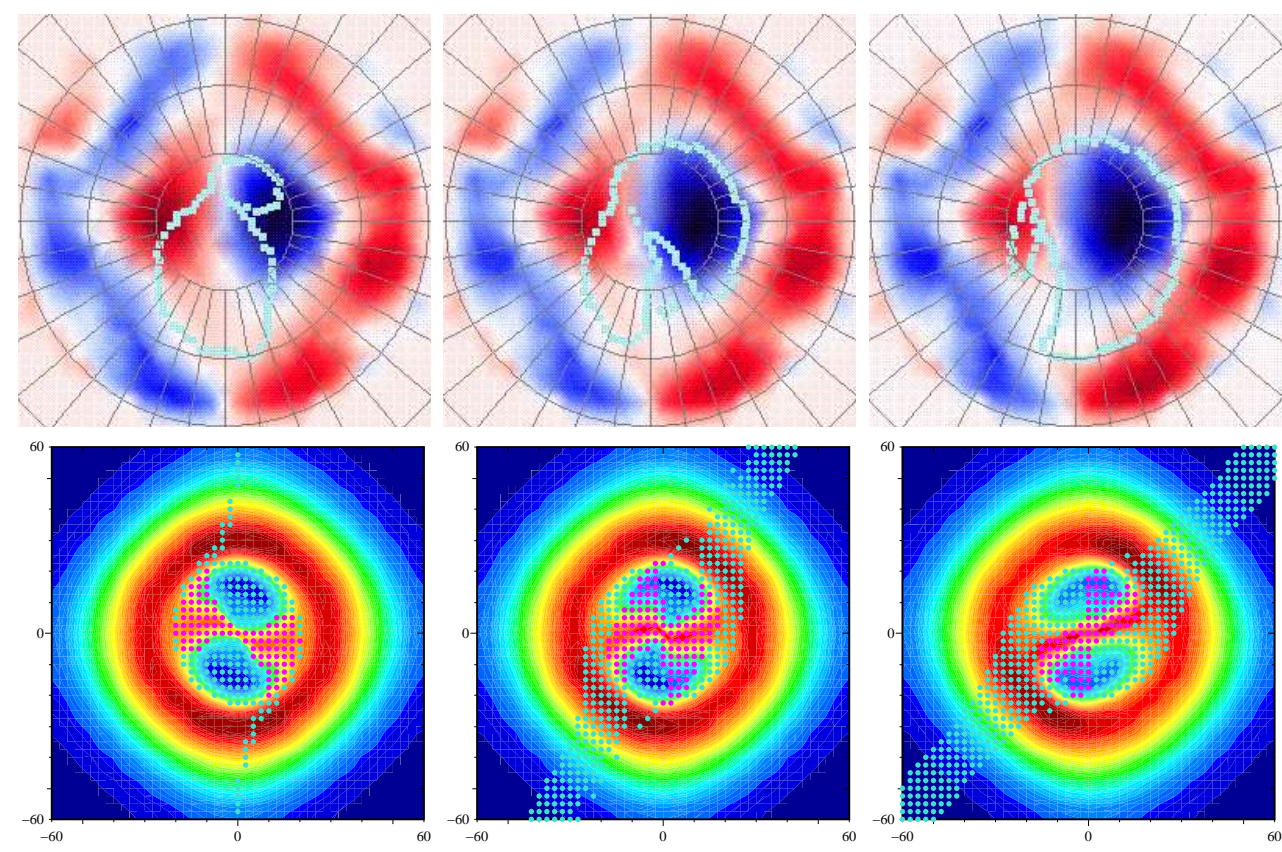

Fig. 10. Simulation results for the MHD run $2 \mathrm{~b}$, with a slow $(1 \mathrm{~h})$ IMF $B_{y}$ sign change from $-5 \mathrm{nT}$ to $+5 \mathrm{nT}$ after two hours constant IMF and constant positive IMF $B_{z}$ of $+5 \mathrm{nT}$. The columns (from left to right) show the state of the magnetosphere 13, 28 and $43 \mathrm{~min}$ after the center of the IMF $B_{y}$ sign-change region has passed the dayside magnetopause. The first row shows the northern ionosphere and the second row shows tail cross sections at $x=-30 R_{E}$. The plots are defined in the same way as in Fig. 1.

angle dependent dawn-duskward width of the far-tail lobes (Raeder et al., 1995) and the north-southward distance between the regions of open field lines in the far tail (Berchem et al., 1998). The qualitative agreement between our simulations and earlier results is good. A quantitative comparison is more difficult. Both data studies and MHD studies report a variety of different values for, for example, the length of the closed field line region, the degree of plasma sheet twisting and the IMF $B_{y}$ penetration rate. Analyzing magnetotail data, it is difficult to determine in which region the measurements were taken, as it takes a long time for a satellite to cross the magnetotail and IMF conditions may change rapidly. The Kaymaz et al. (1994) results of the average tail structure in the xy-plane of the mid-tail may be the most interesting ones, as they are extracted from a large set of IMP-8 data which gives a nearly complete coverage of the tail and surrounding solar wind regions between $15 R_{E}$ and $45 R_{E}$. MHD models often have unrealistically short tail dimensions (e.g. the Fedder-Lyon MHD model). This may be the case for this code as well. It has been proposed by Raeder (1999) that the tail length during northward IMF depends strongly on the rate of numerical diffusion. A high diffusion rate results in a partial decoupling between magnetic flux tube motion and plasma convection, leading to a short closed field line region in the tail (see also the following discussion by Gombosi et al., 2000; Raeder, 2000). Figure 11 shows the $B_{y} B_{z}$ vector components of the total magnetic field in tail cross sections at $x=-10,-15$ and $-30 R_{E}$ after a $2 \mathrm{~h}$ run with a constant pure IMF $B_{y}$ field of $5 \mathrm{nT}$. Compar- ing the model tail shown in Fig. 11 with the average observed tail structure around $33 R_{E}$ for comparable IMF conditions (Kaymaz et al., 1994, Fig. 3c), the model tail resembles the mid-tail observations best for $x=-10 R_{E}$. Further downtail, the IMF dominates the tail structure much more than found by observations. This indicates, that in the present model the length of the closed field line region is too short.

The good agreement between observations, earlier MHD results and this study makes us confident about the significance of the new findings of this study: the relation between the closed field line region in the tail and the shape of the plasma sheet.

\subsection{The IMF $B_{y}$ sign-change cases: comparison with ear-} lier results

The comparison between MHD simulation results with observations by Slinker et al. (2001) shows that moving polar arcs lie indeed on closed field lines, as expected from the finding that particles causing polar arcs originate in the tail plasma sheet (Frank et al., 1986). Therefore, we assume that in our simulations the bridge of closed field lines inside the polar cap can be regarded as the location where a polar arc may occur. For simplicity, the closed field line bridge is referred to further on as "model polar arc". Field-line mapping from the model polar arc to the tail reveals that its source region is in the distant tail, which is in agreement with observations. Large-scale polar arcs are found to have particle characteristics with slightly lower energies than the auroral oval, 
indicating the distant tail as the source region (e.g. Chang et al., 1998). The dawn to dusk motion of the model polar arc in the northern polar cap following an IMF $B_{y}$ sign change from negative to positive during northward IMF is expected from observations (Cumnock et al., 2002). Also the simultaneous occurrence of a polar arc in the Southern Hemisphere but with opposite motion is indicated in data results (Craven et al., 1991). The shape and lifetime of our model polar arcs are quite similar to the model arc shown by Slinker et al. (2001), although their run was done for a stronger and more varying IMF than our runs. Although a more sun-aligned and more homogeneous arc width than seen in the simulations may be expected (Frank et al., 1986), the arc shape is difficult to compare with observations, as there exists a multitude of different moving polar arc shapes (Kullen et al., 2002). A large discrepancy between observations and MHD results is found for the lifetime of moving polar arcs. This is indicated already by Slinker et al. (2001). There, the MSP data shows an arc lifetime of at least two hours, while the model arc persists only for $40 \mathrm{~min}$. The statistical results by Kullen et al. (2002) show that moving polar arcs which cross the entire polar cap exist, on average, for two hours, instead of the $50 \mathrm{~min}$ found in our model study. In single cases, polar arcs can even have lifetimes of three to four hours (see the moving polar arc examples presented by Cumnock et al. (2002) and Kullen et al. (2002)).

The simulation results show a good agreement with the semi-analytical results by Kullen (2000). It confirms the assumption of an IMF $B_{y}$ sign change causing first the nearEarth tail and then the far tail to change its twist such that in an intermediate state the near-Earth and the far tail are oppositely twisted. The main results of the Kullen model are in agreement with the MHD results. The poleward motion of the polar arc, the bifurcation of the closed field line region in the tail, its mapping to the polar arc in the ionosphere, and the non-bifurcation of the plasma sheet occur in both models. The Kullen (2000) model indicates that the width of the sign change region effects the nightside width of the polar arc but not its location. This is confirmed by the MHD simulations as well. However, several IMF $B_{y}$ sign change effects on the magnetosphere appear in the MHD simulations only. The variation of the closed field line region length does not show up in the Kullen (2000) model, as the T89 model used has an open tail. In the Kullen (2000) model the tail twist is a priori forced to change nearly linearly between near-Earth and far tail. The MHD results show that the twist change starts at the flanks and moves within several tens of minutes into the center of the tail (Fig. 5). The polar arc of the MHD simulation stretching from the nightside to the dayside auroral oval and moving over the entire polar cap has a more realistic shape and location than the one presented in the Kullen (2000) model. The suggestion by Chang et al. (1998) that an IMF $B_{y}$ sign change may cause a new polar cap region at prenoon (post-noon) for a sign change of IMF $B_{y}$ from negative to positive (positive to negative) is confirmed by the simulation results. The dawn and duskward displacement of the lobe reconnection in the Northern and Southern Hemisphere,
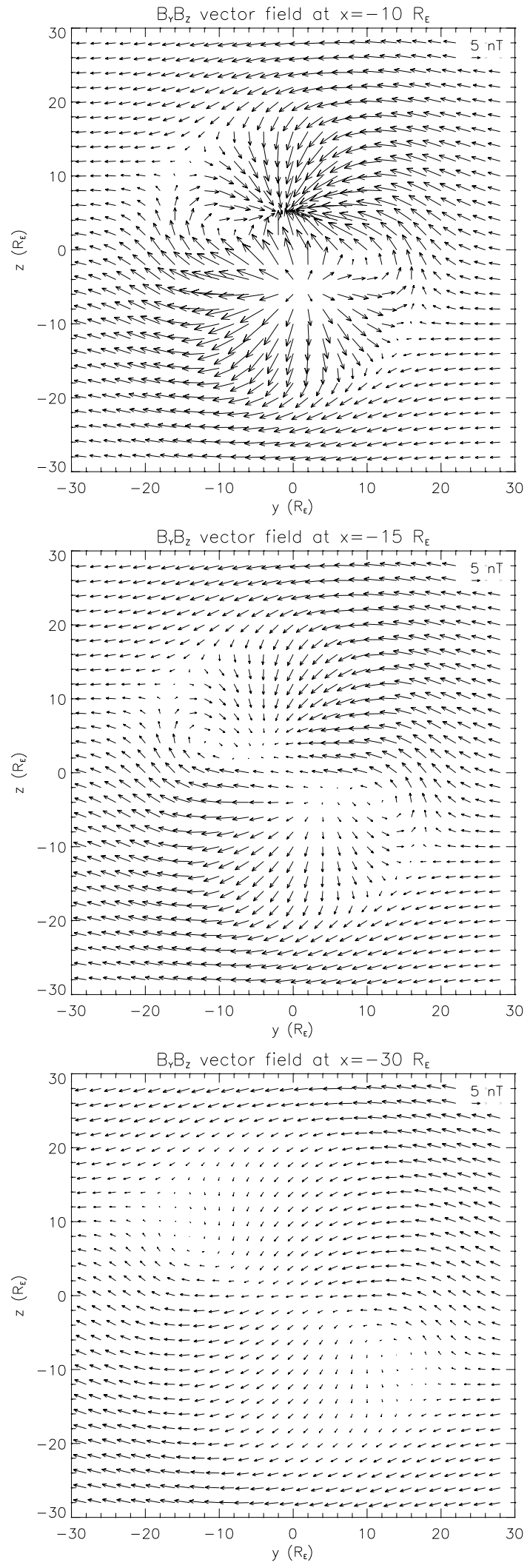

Fig. 11. The total magnetic field at different tail cross sections after two hours constant IMF with an IMF clock angle of $90^{\circ}$ and a magnitude of $5.0 \mathrm{nT}$. 
respectively, changed soon after the IMF $B_{y}$ sign change took place towards the other side of the noon-midnight meridian and is connected to a new polar cap region near noon. However, the dawn to dusk motion of the closed field line bridge in the ionosphere cannot be explained by the dayside reconnection site jump but occurs due to the rotation of the plasma sheet twist, as described by Kullen (2000).

Neither polar arc models, nor the Slinker et al. (2001) MHD results predict the occurrence of solar wind field lines in the mid-tail while a part of the closed field line region extends increasingly tailward. In the GUMICS-4 simulations the region of solar wind field lines inside the tail grows at the expense of the tailward closed field line region until it disappears. Further investigations would be necessary to find out, whether this is a common phenomenon associated with similar IMF configurations. It has to be pointed out, that this process appears at the end of the polar arc lifetime when the arc has already reached the other side of the polar cap and influences neither the creation, nor the dawn-duskward motion of the arc.

3.3 The possible occurrence of polar arcs on high-latitude closed field lines

The suggested appearance of oval-aligned arcs on the strongly poleward displaced dawn side polar cap boundary in run $1 \mathrm{~d}$, the $10^{\circ}$ constant IMF case with dawn-northward IMF, is motivated by observations. Kullen et al. (2002) reported that only the oval-aligned arc type occurs on the dawn (dusk) oval side after one to two hours constant IMF with dominating northward IMF and dawnward (duskward) IMF. Often, oval-aligned arcs are clearly separated from the main oval only during some part of their lifetime, but sometimes they seem to be a part of an extended oval side (e.g. Kullen et al., 2002). Our results are in agreement with the scenario of a strong tail twist being responsible for the poleward displacement of the polar cap (Kullen and Blomberg, 1996) and an oval-aligned arc occurring on its poleward boundary (Meng, 1981). As expected from observations (Cumnock et al., 2002), in runs $2 \mathrm{a}$ and $2 \mathrm{~b}$ a bridge inside the polar cap of closed field lines moves from dawn to dusk after a sign change from dawnward to duskward IMF $B_{y}$, which indicates a moving polar arc. The model polar arc is on both sides clearly separated from the main oval by regions of open field lines, as expected from observations by Huang et al. (1989) and others.

One major concern about the simulation results is that there are no field-aligned currents that are associated with the poleward displaced closed field line region (bridge inside the polar cap) where an oval-aligned (moving) arc is expected to occur. In fact, the FAC current pattern remains nearly unchanged for all runs, while the polar cap boundary changes drastically. The MHD simulations reproduce the large-scale field-aligned current pattern connected to the main auroral oval region fairly well, but small-scale current structures do not appear. This is probably due to limitations of the model, maybe because the grid-resolution in the mag- netotail and in the polar cap is too low. Polar auroral arcs consist of one or several bands of discrete auroral arcs. The associated field-aligned current sheets appear often in (multiple) pairs of upward and downward directed currents, having a width of several tens of kilometers (e.g. Zhu et al., 1997, and references therein). Such small-scale structures cannot be resolved with an effective ionospheric grid resolution of about $300 \mathrm{~km}$. Furthermore, when an upward current sheet is located too close to the return current sheet, no net current will appear in the model. This seems to be a general problem in large-scale magnetosphere MHD models. Even in other MHD models there is a lack of FAC where a bridge of closed field lines appears inside the polar cap (e.g. in the simulation runs for observed polar arcs of Slinker et al. (2001), private communication). Interestingly, in a comparison between auroral images and ionospheric plasma convection by Chang et al. (1998), no plasma convection change associated with a polar arc could be found in SuperDARN measurements, which the authors claim may be due to the small width of the polar arc.

\subsection{The topological connection between polar arcs and the magnetotail}

The MHD simulations show that a small IMF clock angle causes a long tailward extension of the closed field line region and a strong twisting of the far-tail plasma sheet, independent of whether the small clock angle appears for a long time (constant IMF cases) or is in a transitional state (IMF $B_{y}$ sign-change cases). A long closed field line region and a strongly twisted tail causes field lines originating in the far tail to map to high latitudes in the near-Earth region and in the ionosphere. The plasma sheet in the near-Earth tail is only weakly twisted such that the boundary of the closed field line region does not overlap with the plasma sheet boundary but is located in the near-Earth tail lobes.

While the reason for the lack of FAC at the location of a polar arc remains unclear, we believe that the MHD results are correct in the sense that the boundary of the large-scale FAC system does not overlap with the polar cap boundary in all cases. This idea is strengthened by the observational result by Elphinstone et al. (1990), where the main auroral oval (connected to upward FAC) is shown to keep its circular shape even for nonzero IMF $B_{y}$ and positive IMF $B_{z}$, where a strong poleward displacement of the polar cap boundary is expected. We conclude (from mapping FAC to the tail) that the main auroral oval has its source region in the near-Earth region. A polewardly displaced polar cap boundary maps to the highly twisted distant tail and is a possible location for polar arcs to occur.

There are some discrepancies between simulation results and observations. For the constant IMF cases, the most favorable conditions for oval-aligned arcs have been found by observations to occur around 45 degrees, while the simulations suggest a clock angle of around 10 degrees (run 1d). Kullen et al. (2002) showed that during the time period of the statistical study, small clock angles are only slightly less 
common than clock angles of around 45 degrees such that it is not due to the rare occurrence of small clock angles that oval-aligned arcs appear mostly for 45 degrees. In the IMF sign-change cases (run 2a, 2b) the largest difference from the cases is the lifetime of the polar arc. Both deviations from observations may be connected to the unrealistically short tail of the MHD run. A longer region of closed field lines leads to the $B_{y}$ sign reversal taking more time to move tailward, thus the polar arc exists longer. A longer region of closed field lines may lead to that in the $45^{\circ}$ case (run 1c) even far-tail regions with a high twist map to the Earth. This leads to the same effect as in the $10^{\circ}$ case (run 1d): the closed field line region extends into the lobes.

\section{Summary and Conclusions}

Several MHD runs have been performed to examine the influence of constant and changing IMF $B_{y}$ on the large-scale topology of the magnetosphere and the polar ionosphere. The constant IMF runs are done for IMF clock angles of $100^{\circ}, 80^{\circ}, 45^{\circ}$ and $10^{\circ}$ (run 1a-1d). The runs for changing IMF $B_{y}$ consist of a fast (run 2a) and a slow (run 2b) IMF $B_{y}$ sign change during northward IMF.

A dawn-duskward bending of the field lines and a twisting of the tail plasma sheet occur in all constant IMF runs, as expected for nonzero IMF $B_{y}$. Comparing the constant IMF runs for different IMF clock angles reveals that the closed field line region extends the farthest downtail for small clock angles. In that case the downtail increasing twisting of the plasma sheet becomes very high at its tailward end. Field lines originating in the high-latitude part of the far tail map into the lobes of the near-Earth tail. In the ionosphere the polar cap boundary shows a strong poleward displacement on the corresponding oval side. It is suggested that oval-aligned arcs may occur on the strongly poleward displaced boundary of the polar cap. This is in agreement with observations showing that these arcs often appear after hours of nearly constant IMF with a positive $B_{z}$ and a nonzero $B_{y}$ component. The simulation results indicate that oval-aligned arcs map into the highly twisted far-tail plasma sheet, while the main oval maps to the near-Earth and mid-tail regions.

The simulation of an IMF $B_{y}$ sign change during positive IMF $B_{z}$ shows that such a sign reversal does not affect the entire tail at once but starts at the near-Earth tail flanks and then propagates both tailward and toward the tail center. A tailward moving cone of zero $B_{y}$ develops. Inside the cone, the extra $B_{y}$ field and the tail twist are in opposite direction than outside the cone. During the tailward propagation of the sign-change region, the region of closed field lines increases far downtail until it returns to its original length. In an intermediate state the closed field line region is extremely long, with the far-tail plasma sheet and the near-Earth plasma sheet being oppositely twisted. A complicated field line topology emerges. Closed field lines originating in the highly twisted far-tail plasma sheet bifurcate the near-Earth tail lobes and the polar cap. A bridge of closed field lines in the polar cap moves within $50 \mathrm{~min}$ from one oval side to the other. This dawn-duskward moving bridge is interpreted as the location where a moving polar arc may occur. Such a polar arc type appears typically after an IMF $B_{y}$ sign change during predominately northward IMF. A comparison between a fast and a slow IMF $B_{y}$ sign change shows that neither the magnetotail topology, nor the polar arc lifetime and location are significantly different. Only the dawn-dusk width of the closed field line bridge and the length of the closed field line region are influenced by the speed of an IMF $B_{y}$ sign change.

In summary, moving polar arcs and oval-aligned arcs have in common that the IMF clock angle is small, either transitionally (moving polar arcs) or constantly (oval-aligned arcs), and the closed field line region extends far downtail and the plasma sheet is highly twisted at its tailward end. This tail topology causes field lines originating in the highlatitude far-tail region to map high into the near-Earth tail lobes and poleward of the average polar cap boundary. The poleward displaced closed field line region is a probable location for polar arcs to occur.

Acknowledgements. We would like to thank Minna Palmroth for executing the MHD runs.

Topical Editor M. Lester thanks J. Raeder and another referee for their help of evaluating this paper.

\section{References}

Berchem, J., Raeder, J., Ashour-Abdalla, M., Frank, L. A., Peterson, W. R., Ackerson, K. L., Kokubun, S., Yamamoto,T., and Lepping, R. P.: The distant tail at $200 R_{e}$ : Comparison between Geotail observations and the results from a global magnetohydrodynamic simulation, J. Geophys. Res., 103, 9121, 1998.

Borovsky, J. E., Thomsen, M. F., and Elphic, R. C.: The driving of the plasma sheet by the solar wind, J. Geophys. Res., 103, $17617,1998$.

Brecht, S. H., Lyon, J., Fedder, J. A., and Hain, K.: MHD model of IMF By influence on tail, J. Geophys. Res., 103, 17 617, 1981.

Chang, S.-W., Scudder, J. D., Sigwarth, J. B., et al.: A comparison of a model for the theta aurora with observations from Polar, Wind and SuperDARN, J. Geophys. Res., 103, 17 367, 1998.

Cowley, S. W. H.: Magnetospheric asymmetries associated with the y-component of the IMF, Planet. Space Sci., 29, 79, 1981.

Craven, J. D., Murphree, J. S., Frank, L. A., and Cogger, L. L.: Simultaneous optical observations of transpolar arcs in the two polar caps, Geophys. Res. Lett., 18, 2297, 1991.

Cumnock, J. A., Sharber, J. R., Heelis, R. A., Hairston, M. R., and Craven, J. D.: Evolution of the global aurora during positive IMF $B_{z}$ and varying IMF $B_{y}$ conditions, J. Geophys. Res., 102, $17489,1997$.

Cumnock, J. A., Sharber, J. R., Heelis, R. A., Blomberg, L. G., Germany, G. A., Spann, J. F., and Coley, W. R.: Interplanetary magnetic field control of theta aurora development, J. Geophys. Res., 107(A7), 1108, doi:10.1029/2001JA009126, 2002.

Elphinstone, R. D., Jankowska, K., Murphree, J. S., and Cogger, L. L.: The configuration of the auroral distribution for interplanetary magnetic field $B_{z}$ northward, 1. IMF $B_{x}$ and $B_{y}$ dependencies as observed by the Viking satellite, J. Geophys. Res., 95, 5791, 1990.

Fairfield, D. H.: On the average configuration of the geomagnetic tail, J. Geophys. Res., 84, 1950, 1979. 
Fedder, J. A. and Lyon, J. G.: The Earth's magnetosphere is $165 R_{e}$ long: Self-consistent currents, convection, magnetospheric structure, and processes for northward interplanetary magnetic field, J. Geophys. Res., 100, 3623, 1995.

Frank, L. A. and Craven, J. D.: Imaging results from Dynamics Explorer 1, Rev. Geophys., 26, 249, 1988.

Frank, L. A., Craven, J. D., Gurnett, D. A., et al.: The theta aurora, J. Geophys. Res., 91, 3177, 1986.

Gombosi, T. I., DeZeeuw, D. L., Häberli, R. M., Powell, K. G., and Song, P.: The length of the magnetotail for northward IMF: Results of 3-D MHD simulations, in: Physics of Space Plasmas, edited by Chang, T. and Jasperse, J. R., 15, 121, MIT Press, Cambridge, 1998 .

Gombosi, T. I., Powell, K. G., and van Leer, B.: Comment on "Modeling the magnetosphere for northward interplanetary magnetic field: Effects of electrical resistivity" by Raeder, J., J. Geophys. Res., 105, 13 149, 2000.

Huang, C. Y., Craven, J. D., and Frank, L. A.: Simultaneous observations of a theta aurora and associated magnetotail plasmas, J. Geophys. Res., 94, 10 137, 1989.

Janhunen, P.: GUMICS-3 - A global ionosphere-magnetosphere coupling simulation with high ionopheric resolution, ESA Sympos. Proc., SP-392, 233, 1996.

Janhunen, P. and Huuskonen, A.: A numerical ionospheremagnetosphere coupling model with variable conductivities, J. Geophys. Res., 98, 9519, 1993.

Janhunen, P. and Palmroth, M.: Some observational phenomena are well reproduced by our global MHD while others are not: Remarks on what, why and how, Adv. Space Res., 28, 1685, 2001.

Kan, J. R. and Burke, W. J.: A theoretical model of polar cap auroral arcs, J. Geophys. Res., 90, 4171, 1985.

Kaymaz, Z. and Siscoe, G.: Open geometry of the magnetotail cross section, J. Geophys. Res., 103, 14 829, 1998.

Kaymaz, Z., Siscoe, G. L., Luhmann, J. G., Lepping, R. P., and Russell, Ch. T.: Interplanetary magnetic field control of magnetotail magnetic field geometry: IMP 8 observations, J. Geophys. Res., 99, $11113,1994$.

Kaymaz, Z., Siscoe, G. L., Luhmann, J. G., Fedder, J. A., and Lyon, J. G.: Interplanetary magnetic field control of magnetotail field IMP-8 data and MHD model compared, J. Geophys. Res., 100, $17163,1995$.

Kullen, A.: The connection between transpolar arcs and magnetotail rotation, Geophys. Res. Lett., 27, 73, 2000.

Kullen, A. and Blomberg, L. G.: The influence of IMF $B_{y}$ on the mapping between the Earth's magnetotail and its ionosphere, Geophys. Res. Lett., 23, 256, 1996.

Kullen, A., Brittnacher, M., Cumnock, J. A., and Blomberg, L. G.: Solar wind dependence of the occurrence and motion of polar auroral arcs: A statistical study, J. Geophys. Res., 107(A11), 1362, doi:10.1029/2002JA009245, 2002.

Lui, A. T. Y.: Polar wind influence on magnetotail configuration and dynamics, in: Solar wind magnetosphere coupling, edited by Kamide, Y. and Slavin, J. A., 671, 1986.

MacWan, S. E.: A determination of twisting of the Earth's magnetotail at distances $115-220 R_{e}$ : ISEE 3, J. Geophys. Res., 97, 19.239, 1992.

Makita, K., Meng, C. I., and Akasofu, S. I.: Latitudinal electron precipitation patterns during large and small IMF magnitudes for northward IMF conditions, J. Geophys. Res., 93, 97, 1988.

Meng, C.-I.: Polar cap arcs and the plasma sheet, J. Geophys. Res., $8,273,1981$.
Newell, P. T., Xu, D., Meng, C.-I., and Kivelson, M. G.: Dynamical polar cap: A unifying approach, J. Geophys. Res., 102, 127, 1997.

Ogino, T.: A three dimensional MHD simulation of the interaction of the solar wind with the Earth's magnetosphere: The generation of field aligned currents, J. Geophys. Res., 91, 6791, 1986.

Ogino, T., Walker, R. J., and Ashour-Abdalla, M.: A global magnetohydrodynamic simulation of the magnetosheath and magnetosphere when the interplanetary magnetic field is northward, IEEE Trans. Plasma Sci., 20, 6, 817, 1992.

Owen, C. J., Slavin, J. A., Richardson, I. G., Murphy, N., and Hynds, R. J.: Average motion, structure and orientation of the distant magnetotail determined from remote sensing of the edge of the plasma sheet boundary layer with $E>35 \mathrm{keV}$ ions, J. Geophys. Res., 100, 185, 1995.

Raeder, J.: Modeling the magnetosphere for northward interplanetary magnetic field: Effects of electrical resistivity, J. Geophys. Res., 104, 17 357, 1999.

Raeder, J.: Comment on "Modeling the magnetosphere for northward interplanetary magnetic field: Effects of electrical resistivity" by Raeder, J. - Reply, J. Geophys. Res., 105, 13 149, 2000.

Raeder, J., Walker, R. J., and Ashour-Abdalla, M.: The structure of the distant geomagnetic tail during long periods of northward IMF, Geophys. Res. Lett., 22, 349, 1995.

Rezhenov, B. V.: A possible mechanism for theta aurora formation, Ann. Geophys., 13, 698, 1995.

Russell, C. T.: The configuration of the magnetosphere, in: Critical Problems of Magnetospheric Physics, edited Dyer, E. R., National Academy of Sciences, Washington, D. C., 1, 1972.

Sergeev, V. A.: Penetration of the By-component of the interplanetary magnetic field (IMF) into the tail of the magnetosphere, Geomagn. Aeronom., 27, 4, 1987.

Sibeck, D. G., Slavin, J. A., Smith, E. J., and Tsurutani, B. T.: Twisting of the geomagnetic tail, in: Solar Wind-Magnetosphere Coupling, edited by Kamide, Y. and Slavin, L. A., 731, 1986.

Slinker, S. P., Fedder, J. A., McEwen, D. J., Zhang, Y., and Lyon, J. G.: Polar cap study during northward interplanetary magnetic field on 19 January 1998, Physics of Plasmas, 8, 1119, 2001.

Song, P., DeZeeuw, D. L., Gombosi, T. I., Groth, C. P. T., and Powell, K. G.: A numerical study of solar wind-magnetosphere interaction for northward interplanetary magnetic field, J. Geophys. Res., 104, 28 361, 1999.

Tanaka, T.: Configuration of the magnetosphere-ionosphere convection system under northward IMF conditions with nonzero IMF $B_{y}$, J. Geophys. Res., 10, $14683,1999$.

Tsyganenko, N. A.: A magnetospheric magnetic field model with a warped tail current sheet, Planet. Space Sci., 37, 5, 1989.

Tsyganenko, N. A.: Global configuration of the magnetotail current sheet as derived from Geotail, Wind, IMP 8 and ISEE 1/2 data, J. Geophys. Res., 103, 6827, 1998.

Valladares, C. E., Carlson Jr., H. C., and Fukui, K.: Interplanetary magnetic field dependency of stable Sun-aligned polar cap arcs, J. Geophys. Res., 99, 6247, 1994.

Voigt, G.-H. and Hilmer, R. V. : The influence of the IMF $B_{y}$ component on the Earth's magneto-hydrostatic magnetotail, in: Magnetotail physics, edited by Lui, A. T. Y., Laurel Maryland, 1987.

Walker R. J., Richard, R. L., Ogino, T., and Ashour-Abdalla, M.: The response of the magnetotail to changes in the IMF orientation: The magnetotail's long memory, Phys. Chem. Earth. Pt. C, 2, 221, 1999.

Zhu L., Schunk, R. W., and Sojka, J. J.: Polar cap arcs: a review, J. Atmos. and Solar-terrest. Phys., 59, 1087, 1997. 